Hydroécol. Appl. (1996) Tome 7 Vol. 1-2, pp. 151-183

\title{
Les berges du fleuve Sinnamary (Guyane française) : géomorphologie et groupements végétaux
}

\author{
The banks from Sinnamary river (french Guiana): \\ geomorphology and vegetation
}

\author{
Michel Hoff \\ ORSTOM, Service du Patrimoine Naturel, I.E.G.B. M.N.H.N., 57 rue Cuvier, F-75005 Paris.
}

Résumé. - Les groupements végétaux liés au cours moyen du Sinnamary, plus particulièrement au niveau des sauts, sont remarquables par leur diversité. Ils ont en grande partie disparu à la suite de la mise en eau du barrage de Petit-Saut. C'est pourquoi la géomorphologie des principaux sauts est décrite, ainsi que les formations végétales ripicoles. Les groupements marécageux, forestiers ou arbustifs, sont typiques pour la Guyane. II en est de même pour les franges ripicoles des forêts. Par contre, la forêt dense de basse altitude est moins originale et ne diffère pas fondamentalement de celle des bassins versants voisins. Les groupements de berges sont cartographiés entre Saut Vata et Saut Stéphanie, ce qui permet de décrire différents types d'organisation végétale le long du Sinnamary.

Mots-clés. - Fleuve, Saut, Végétation ripicole, Forêt primaire, Guyane, Région équatoriale

Abstract. - The vegetation of the middle reaches of the Sinnamary river is remarquable. The hydroelectric reservoir will run $80 \mathrm{~km}$ down the river. The geomorphology of the most important rapids and the river vegetation are described. The swamp shrub, the swamp and ripicolous forests are typical. The river vegetation between Saut Vata and Saut Stéphanie is mapped. Several vegetation types are described, based on floristics and structure.

Key words. - River, Rapid, Ripicolous vegetation, Primary rain forest, French Guiana, Equatorial region 


\section{INTRODUCTION}

La bassin du Sinnamary s'étend sur plus de $6000 \mathrm{~km}^{2}$ dans la partie nord de la Guyane. La flore est encore imparfaitement connue car seules 1212 espèces y ont été recensées. Des zones écologiquement comparables, mieux prospectées (région de Cayenne, Bas-Oyapock - Crique Gabaret, etc.), présentent une richesse floristique de l'ordre de 1500 espèces, soit un peu plus du quart de la flore de Guyane (Cremers et al., 1991). Cette relative pauvreté du bassin du Sinnamary est due à l'absence de relief important et de groupements ouverts tels que les savanes et les formations liées aux inselbergs.

La flore, la structure et la dynamique des végétations ripicoles, notamment au niveau des chutes, sont dans l'ensemble, peu étudiées au niveau équatorial. Léonard (1993) présente néanmoins une synthèse pour les chutes de la Tshopo au Zaïre, Traore et al. (1990) étudient les lac de barrage de Côte d'Ivoire. Lamotte (1992), décrit la dynamique des forêts inondables de la Haute-Amazonie au Pérou. Cependant la largeur des milieux ripicoles en Guyane est infime par rapport à l'Amazonie (quelques dizaines de mètres au niveau des grands sauts au lieu de plus de $2 \mathrm{~km}$ au Pérou).

Même pour les Guyanes, la végétation associée directement aux cours des rivières a été assez peu étudiée (Van Donselaar, 1989; Oldeman, 1972).
La note présentée est le résultat des travaux effectués dans le cadre de l'étude d'environnement ORSTOM - EDF préalable à la mise en eau du barrage de Petif-Saut (Cerdan, 1993; Hoff, 1993; Lauzanne et al., 1993).

Après une présentation du milieu physique, la géomorphologie des sauts puis la flore et de l'écologie des groupements végétaux liées aux berges sont décrits. Le Sinnamary, fleuve de taille moyenne, présente une zonation sud-nord dont la partie amont est traitée ici. La région considérée au point de vue botanique, s'étend sur tout le moyen Sinnamary, à partir de Saut Vata et Saut Kawènn, jusqu'à Saut Parasol. La partie du bassin comprise entre Petit-Saut et Saut Takari-Tanté est désormais recouvert par une retenue hydroélectrique d'environ $310 \mathrm{~km}^{2}$ (Sissakian, 1992).

La zone étudiée couvre la totalité du bassin du Sinnamary, soit environ $6000 \mathrm{~km}^{2}$ (Carte 1), sauf la zone littorale jusqu'à Crique Vénus. Elle est comprise entre $52^{\circ} 45^{\prime}$ et $53^{\circ} 20^{\prime}$ ouest d'une part et entre $4^{\circ}$ et $5^{\circ} 12^{\prime}$ nord d'autre part.

\section{LE MILIEU PHYSIQUE}

\subsection{Climatologie et géologie}

La Guyane se situe sur le continent sud-américain et couvre une superficie d'environ $90000 \mathrm{~km}^{2}$ comprise entre $2^{\circ}$ et $5^{\circ}$ de latitude Nord et $52^{\circ}$ et $54^{\circ}$ de longitude Ouest. Son climat est 


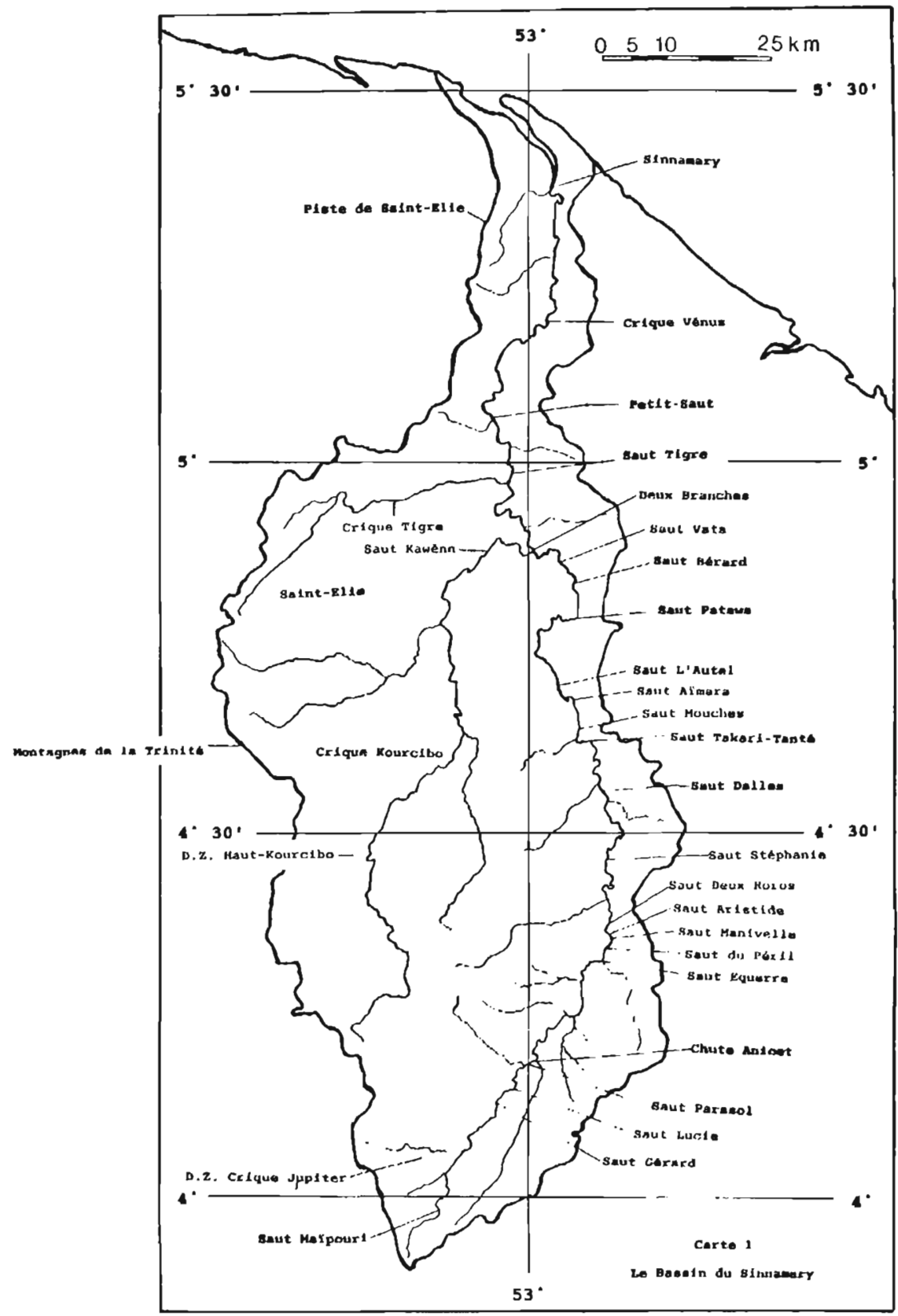

Carte 1. - Le Bassin du Sinnamary.

Map 1. - The Sinnamary basin. 
de type équatorial (Boyé et al., 1979); la température moyenne annuelle, calculée sur 24 ans, est de $26^{\circ} \mathrm{C}$, les maxima et minima étant respectivement 21 et $31^{\circ} \mathrm{C}$; les précipitations sont abondantes (entre 2000 et $4000 \mathrm{~mm}$ ); l'humidité de l'air est comprise entre 80 et $90 \%$.

Dans l'ensemble, le bassin du Sinnamary est situé dans la région climatique II $b$ (Planche 6. Atlas de Guyane). Celle-ci se caractérise par un climat relativement régulier, " avec des pluies d'intensité en moyenne modérée, des vents faibles et une évaporation faible ". La grande saison sèche (de juillet à novembre) est plus courte que dans le bassin voisin de l'Approuague, mais le "petit été de février-mars", qui correspond à une petite saison sèche, y est bien marqué.

Le bassin du Sinnamary est situé en grande partie au-dessus de l'isohyète 3000 avec une pointe à 3600 en aval du site de Petit Saut (Crique Grégoire) et un minimum frôlant l'isohyète 2000 dans le haut Kourcibo.

Le site du barrage se trouve sur des terrains métamorphiques antécambriens de la série de l'Orapu. Plus en amont, la rivière traverse le Paramaca inférieur et supérieur au niveau de Saut Tigre et la série de Borodino à Saut Vata. Le fleuve méandre ensuite sur des migmatites, des gneiss, des granites et des diorites dont les filons affleurants sont responsables des nombreux sauts. Saut l'Autel, par exemple, correspond à des gneiss et des granites guyanais, Saut Dalles à des migmatites, Saut Parasol à des diorites quarztiques et Saut Maïpouri à des gneiss et des granites guyanais. Des filons de dolérites sillonnent longitudinalement tout le bassin.

\subsection{Hydrologie}

Le Sinnamary est le cinquième fleuve de Guyane par la superficie de son bassin et le quatrième par son débit. II présente, comme la plupart des fleuves et des rivières de Guyane, une orientation sud-nord. II prend sa source dans les montagnes du nord de Saül, château d'eau de la Guyane. Mais le bassin du Sinnamary est également alimenté par des nombreux châteaux d'eau secondaires, comme les Montagnes de la Trinité pour le Kourcibo, la Montagne des Trois Roros pour la crique Tigre. L'ensemble du bassin du Sinnamary (Carte 1) est asymétrique, réduit du côté est. Le cours principal du fleuve y est souvent à moins de $10 \mathrm{~km}$ du bassin du Kourou, de la Comté ou de l'Approuague. Le bassin, par contre, s'étale largement à l'ouest, sur plus de $50 \mathrm{~km}$. En aval, il passe par un étroit goulet de $10 \mathrm{~km}$ de large, entre la crique Yiyi et la crique Paracou, avant de se jeter dans I'Océan Atlantique.

Les surfaces des principales parties du bassin du Sinnamary (Hiez et al., 1964) sont présentées dans le tableau 1. Au niveau du site du barrage, à Petit-Saut, le Sinnamary draine un bassin de $5927 \mathrm{~km}^{2}$. La limite et la surface exacte du bassin sont cependant imprécises car les cols sont souvent occupés par des forêts plus ou moins marécageuses 
Tableau 1. - Superticie en km des principaux bassins versants du Sinnamary (d'après Hiez et Dubreuil, 1964 et Laboratoire d'Hydrologie, centre ORSTOM de Cayenne 1992).

Table 1. - Area of the main Sinnamary sub-basins.

\begin{tabular}{|c|c|c|c|}
\hline Localité & \multicolumn{2}{|c|}{ Surface du Bassin } & Surface cumulée \\
\hline & Cours principal & Affluents & \\
\hline $\begin{array}{l}\text { Takari-Tanté } \\
\text { Saut I'Autel } \\
\text { Deux Branches }\end{array}$ & $\begin{array}{r}2065 \\
135 \\
365\end{array}$ & & $\begin{array}{l}2065 \\
2200 \\
2565\end{array}$ \\
\hline Kourcibo & & 2350 & 4915 \\
\hline Confluent Crique Tigre & 205 & & 5120 \\
\hline |Crique Tigre & & 558 & \\
\hline |Crique Aïmara & & 120 & \\
\hline Petit-Saut & 129 & & 5927 \\
\hline Crique Grégoire & & 12 & \\
\hline $\begin{array}{l}\text { Village Sinnamary } \\
\text { Embouchure }\end{array}$ & $\begin{array}{r}700 \\
40 \\
\end{array}$ & & $\begin{array}{l}6639 \\
6679\end{array}$ \\
\hline
\end{tabular}

d'assez grandes surfaces qui communiquent avec les bassins voisins.

Le régime hydrologique présente deux périodes de hautes eaux, en janvier et en mai-juin. La caractéristique principale de ce régime hydrique est liée à la rapidité de la montée du niveau des eaux lors des fortes pluies. En effet, le pouvoir de rétention des sols est faible. Cependant, le ruissellement direct est dans l'ensemble faible car l'eau s'infiltre en immense majorité. Les débits à A-Dieu-Vat vont de 25 à $200 \mathrm{~m}^{3} / \mathrm{s}$ avec probablement des crues à $300 \mathrm{~m}^{3} / \mathrm{s}$. Le débit moyen annuel est de $275 \mathrm{~m}^{3} / \mathrm{s}$ avec une crue vingtenale de $1500 \mathrm{~m}^{3} / \mathrm{s}$.

\section{GÉOMORPHOLOGIE DES SAUTS ET DES BERGES}

Le cours du Sinnamary présente des particularités morphologiques qu'il convient de décrire. Nous nous attacherons plus particulièrement aux sauts. Une vingtaine de sauts ou de formes de berges ont été dessinés sur le terrain, puis repris à partir des photos aériennes lorsqu'elles étaient disponibles. Les cartes détaillées sont numérotés de 1 à 22 de l'aval vers l'amont. Les chiffres entre parenthèses après le nom du saut dans le texte suivant indiquent le numéro de la figure correspondante. 


\subsection{Les sauts}

La présence de sauts, chutes et rapides de taille et de morphologie variables est l'une des particularités des rivières de Guyane. Ils sont généralement dus à des filons de roches saines après affouillement des altèrites. Les principaux sauts se trouvent sur des filons de dolérites, roches plus dures que les schistes, gneiss, granites et migmatites voisins. Ces filons ont une direction générale NNW-SSE. Leur largeur est variable, de quelques centimètres à plusieurs centaines de mètres. Les principaux sauts dus aux dolérites sont les suivants: Petit-Saut, Saut Vata et Saut Kawènn, Saut Bérard, Saut Patawa, tous les sauts entre Saut Nacibo et Saut Takari-Tanté - la rivière serpente d'ailleurs le long de la ligne de dolérites - et les sauts entre Saut Deux Roros et Saut Equerre. Par contre d'autres sauts ne sont pas dus aux dolérites, Saut Tigre, Saut Limonet, Saut Parole-Finie, Saut Stéphanie, Saut Parasol et tous les sauts en amont de Saut Parasol. Les sauts en aval de Saut Vata sont plutôt des complexes plus ou moins larges de rapides sans réelle rupture de pente.

On peut observer le faible nombre des chutes, avec rupture de charge, par rapport aux sauts et rapides.

Les sauts peuvent se regrouper en deux grands groupes, les sauts étroits et les sauts larges. La présence de forêts inondables ou de groupements herbacés et arbustifs marécageux, appelés "pripris" en Guyane, en aval ou en amont et d'îles ou d'îlots plus ou moins importants caractérisent des sous groupes. La présence d'un chenal latéral secondaire, appelé "bistouri » en Guyane, de taille réduite par rapport au chenal principal, est un élément typologique important.

La végétation citée au niveau des sauts est décrite en seconde partie.

\subsubsection{Les sauts étroits}

\subsubsection{Les sauts étroits sans bistou- ri : Saut Mouche}

Les sauts étroits se caractérisent par un goulet d'étranglement aussi large ou moins large que le lit mineur du Sinnamary en amont ou en aval du saut. Ils sont de longueur variable et présentent une ou plusieurs marches. Les forêts inondables sont absentes ou de faible largeur. Les pripris sont en aval de la chute, au niveau d'un élargissement du lit mineur. Des bancs de sables peuvent être également présents en aval.

Les sauts suivants sont de ce type : Saut l'Autel (carte détaillée 4), saut Mouche (7), Saut Dalles (9), la première marche de Saut Manivelle (16) et la Chute Anicet (19).

\subsubsection{Les sauts étroits avec bistou- ri : Saut Parasol}

Ces sauts sont peu nombreux, et de morphologie très complexe. Les forêts inondables et les pripris sont souvent importants en aval. Les îlots de saut peuvent être très importants. Les sauts principaux sont: Saut Vata, Saut Takari-Tanté (carte détaillée 8) et Saut Parasol (20). 


\subsubsection{Les sauts larges}

Au niveau du saut, on observe un élargissement important de la rivière. Les rapides sont souvent en éventail, avec ou sans îlots ou îles centrales. La plupart de ces sauts présentent des structures de saut étroit dans leur partie en aval.

\subsubsection{Les sauts larges rocheux: Saut Equerre}

Le saut n'est constitué que de plaques rocheuses de tailles variables. Selon son degré d'évolution, l'éventail est plus ou moins complet. Les pripris et forêts inondables, s'ils sont présents, sont toujours en aval de la chute. Exemples: deux sauts sans nom entre Saut Stéphanie et Saut Deux Roros (cartes détaillées 11 et 12), Saut Deux Roros 1 (13) et Saut Equerre (18).

\subsubsection{Les sauts larges à ilots : Saut du Péril}

Ce type de sauts est probablement le plus intéressant au point de vue paysager. Les chutes se caractérisent par un fort élargissement de la rivière au niveau de la rupture. Les bancs de rochers et les ilots avec une végétation ligneuse sont nombreux. Ces îlots présentent une flore particulière car il s'agit d'un des seuls groupements ligneux bas et ouverts de l'intérieur de la Guyane (si l'on excepte les forêts basses sur inselberg). Nous y reviendrons dans la partie consacrée à la végétation. Les forêts inondables et les pripris se trouvent en amont de la chute. Celle-ci est très souvent en plu- sieurs marches. Seul le Saut du Péril présente un bistouri permanent.

Ces sauts sont les plus nombreux: Saut Deux Roros 2 (carte détaillée 14), Saut Aristide (15), Saut Manivelle (= Saut Alcide) (16), Saut du Péril (17), Saut Lucie (21), Saut Gérard (22).

3.1.2.3 Les sauts larges à iles centrales : Saut Patawa

Ce sont les plus grands sauts du Sinnamary. L'ensemble peut atteindre $200 \mathrm{~m}$, voire $300 \mathrm{~m}$ de longueur. Au milieu du saut, une île de taille importante est recouverte par une forêt de même type que la forêt environnante. Généralement, une grande forêt inondable se trouve en aval du saut ainsi que des pripris et parfois une forêt marécageuse.

Les principaux sauts de ce type sont Saut Bérard (carte détaillée 2), Saut Patawa (3), Saut Koumarou (5) et Saut Aïmara (6).

\subsubsection{Conclusion}

Peut-on présenter une évolution dynamique dans la structure des sauts? Les sauts larges à îles se trouvent plutôt dans le cours inférieur, les sauts larges à îlots dans le cours supérieur, les sauts larges à bancs de rochers également dans le cours supérieur, mais en amont ou en aval des sauts précédents. Les sauts étroits sans bistouri sont dans le cours moyen ainsi qu'une partie des sauts étroits à bistouri.

II est donc tentant de voir plusieurs complexes de sauts, avec une évolu- 
tion différentielle selon leurs positions. Le Sinnamary présente au moins deux complexes importants de sauts. Le premier va de Saut Bérard à Saut Takari-Tanté, avec en aval des sauts larges à îles forestières et en amont des sauts étroits. Le second complexe débute à Saut Deux-Roros et se termine à Saut Parasol, les premiers sauts présentent des îlots et ceux de l'amont sont étroits. Un troisième complexe occupe probablement le haut-Sinnamary, mais nous n'avons pu étudier tous les sauts en détail, et le dernier saut - Saut Maïpouri n'a pas été atteint. En aval, un complexe débute probablement après Petit-Saut et se termine à Saut Vata, avec des sauts larges et de nombreux groupes de rapides autour de l'île $\mathrm{Ba}$ gatelle et de Saut Tigre, par exemple. Une étude plus précise, qui n'a pas été réalisée avant la mise en eau, auraient permis de compléter la description des sauts et de mieux cerner leur typologie.

\subsection{Les berges}

D'après Cailleux (1959), la vallée d'un cours d'eau en région amazonienne comporte quatre éléments déterminant sa topographie :

- Le versant (zone non ou très rarement inondable)

- Le flat (la plaine d'inondation)

- La berge vive (qui délimite le lit mineur)

- Le lit mineur

Dans certains cas, le flat disparaît et le versant est en contact direct avec la berge vive. Dans ce cas, nous avons une forêt ripicole proprement dite. Dans d'autres cas, la plaine d'inondation est plus basse que le lit mineur (en arrière d'un bourrelet alluvial), ou présente une nappe perchée permanente. Dans ce cas, nous avons une forêt marécageuse.

L'évolution des berges vives (c'està-dire dans le cas ou il n'y a pas de flat) se fait par sapement à la base, puis effondrement en loupe. Ces éboulements sont de l'ordre de 2 à $3 \mathrm{~m}$ pour $100 \mathrm{~m}$, ce qui conduit à un renouvellement général des berges et de la frange ripicole proprement dite de l'ordre de 40 ans.

\subsection{Les méandres}

Choubert (1959), signale la présence de méandres instables et innombrables dans les cours supérieurs des fleuves. Cette vision nous paraît exagérée. S'il y a effectivement des modifications du cours des rivières, celles-ci sont progressives (ou liées à un évènement majeur comme une très forte crue), sauf peut-être dans quelques zones très limitées.

Deux structures de méandres ont été étudiées en détail, la crique Vénus (carte détaillée 1) et Saut Stéphanie (10). Par rapport aux sauts, il faut noter la très grande importance des forêts inondables à Crique Vénus et des forêts inondées et marécageuses et des pripris à Saut Stéphanie. Ce dernier site est particulièrement intéressant car il s'agit d'une vaste zone de marécage boisé située loin de tout au- 
tre saut. Les modifications du cours de la rivière doivent être fréquentes dans cette zone, comme le montre la difficulté de suivre son chemin sur les cartes IGN. Des épingles à cheveux doivent se faire et se défaire lors des très fortes crues. La connaissance de la dynamique de ces chenaux présente une grande importance car elle pourrait permettre de recréer des berges artificielles aux fonds des talweg soumis aux variations du niveau des eaux du barrage.

\section{LA VÉGÉTATION}

\subsection{Méthode d'étude et répartition des observations}

\subsubsection{Méthode}

L'étude écologique du bassin du Sinnamary est réalisée d'une part par des prospections et observations de terrain et des récoltes de plantes et d'autre part par l'exploitation des données recueillies dans la banque de données AUBLET (Hoff et al., 1989), sur la Guyane. Les résultats sont ensuite complétés et généralisés par l'utilisation des photos aériennes et l'interprétation des images satellitaires.

Le recueil des informations se réalise d'abord par des prospections de terrain et un inventaire des formations végétales de toute la zone. Cependant, les observations et la récolte de spécimens d'herbier n'ont pas pu se faire d'une manière systématique dans tout le bassin du Sinnamary, étant donné l'importance de l'aire d'étude et l'inaccessibilité de certaines régions.

Dans la mesure du possible, toutes les plantes en fleur ou en cours de fructification ont été récoltées. Chaque récolte fournit des informations qui ont été introduites dans la base de données ALIBLET. Ces informations sont, principalement: le nom de l'espèce, la localité avec ses coordonnées géographiques, la date, l'altitude, la formation végétale de collecte, la hauteur de la plante et son type biologique, le collecteur, le numéro de collecte, l'herbier de dépôt, etc.

La banque de données "AUBLET " rassemble actuellement environ 117000 spécimens d'herbier de Guyane française représentant plus de 5000 espèces différentes récoltés dans 2000 localités différentes par près de 600 botanistes depuis 1722 .

La caractérisation des formations végétales a été réalisée sur la base des plantes récoltées, et à l'aide des références suivantes: de Granville, (1978, 1986 et 1991), Hoff et al., (1990), Hoff, (1991), Lindeman (1953).

Les algues ne sont pas étudiées ici, voir Champeau, 1991, et Couté (en préparation).

\subsubsection{Répartition des observations}

Dans le bassin du Sinnamary, qui représente un peu moins de $7 \%$ de la Guyane, 6147 spécimens d'herbier ont été récoltés dans 82 localités différentes. Globalement, le bassin du 
Sinnamary commence à être assez bien connu, car avec plus de 6000 récoltes botaniques il est maintenant au niveau de connaissance d'autres régions de Guyane (lle de Cayenne, Piste de Saint-Elie, Arataye-Nouragues, etc.).

Parmi ces récoltes, seuls 3802 spécimens sont déterminés au niveau de l'espèce, soit $62 \%$. En effet, beaucoup de spécimens sont des récoltes récentes (1990-1991) et sont en cours de détermination ou de vérification par les spécialistes des familles concernées.

\subsubsection{Répartition par collecteur et par année}

Au total, 41 botanistes ont fait des observations sur le Sinnamary. La majeure partie de ces récoltes ont été faites dans le cadre des études relatives à l'environnement du barrage de Petit-Saut (étude d'impact comprise). II s'agit des récoltes Hoff et al. (1879 spécimens collectés), Loubry (810 spécimens), Bordenave (652), Granville et al. (512), Prévost \& Sabatier (318), Mori et al. (315), Pasch (123), soit au total 4609 spécimens $(75 \%)$.

Très peu de récoltes ont été effectuées avant 1965 (Aublet - 17631764, Leblond, 1788, Gabriel, 1802 et Leprieur, 1837-1839) et, de plus, elles ne sont pas localisées avec précision. Les collectes récentes ont débuté en 1965. Jusqu'à 1979, 1138 spécimens sont récoltés, soit moins de $20 \%$ du total. L'époque concernant et suivant l'étude d'impact (1983-1987) est pauvre en récoltes (197 spécimens, à peine $3 \%$ ). L'inventaire floristique du
Sinnamary a débuté réellement en 1988. Près de $75 \%$ des spécimens d'herbier ont été prélevés entre 1989 et 1994 .

\subsubsection{Répartition par localité et par formation végétale}

Les principales localités de collectes sont les suivantes: Crique et Montagne Plomb (982 spécimens), Site de Petit Saut - Barrage et environs (774), Crique Grégoire (548), Zone d'atterrissage pour hélicoptère (appelée D.Z. ou Dropping Zone) de Saut Dalles et environs (410), D.Z. de Crique Jupiter et environs (363), Saut Takari-Tanté (326), D.Z. de Saut Aïmara (268) D.Z. du Haut-Kourcibo (229), Saut Kawènn - Crique Kourcibo (196), Saut Parasol (182), Saut Stéphanie (175).

La répartition des spécimens par localité montre nettement les faibles récoltes au niveau des sauts du moyen Sinnamary, de Saut Vata à Saut Takari-Tanté et au dessus de Saut Stéphanie (mis à part Saut $\mathrm{Pa}$ rasol). Ces lacunes ont été partiellement comblées lors d'une mission en janvier 1992. Le Kourcibo est pratiquement inconnu, même dans la partie qui va être submergée.

Les échantillons ont été récoltés dans 64 groupements végétaux différents. Les espèces ont été regroupées dans 13 formations végétales principales.

Le moyen et le haut-Sinnamary ne présentent pas de groupements végétaux aquatiques ou semi-aquatiques ouverts à eau dormante ou stagnante, de surface notable. Ces groupements 
occupent de très grandes surfaces dans toute la zone littorale (Bonnet et al., 1988; Champeau et al., 1991; Dessillons et al., 1989) où ils sont remarquablement diversifiés (Hoff et al., 1993).

\subsection{Description des groupements végétaux}

\subsubsection{Les groupements ouverts}

La liste des espèces des milieux ouverts est donnée en annexe 1.

\subsubsection{Les groupements ouverts}

\subsection{Les groupements aquatiques}

Les aquatiques macrophytes (c'est-àdire les plantes qui effectuent la totalité de leur cycle de reproduction dans ou sur l'eau) sont très rares, voire totalement absentes dans le secteur étudié (Champeau, 1991). Au Surinam, Werkhoven (1993) dénombre 68 espèces de macrophytes qui ne sont présentes, mise à part les podostemonacées, qu'en plaine côtière et en savane. La turbidité de l'eau, la profondeur du lit ainsi que la rapidité du courant, spécialement lors des crues, empêchent le développement de végétation aquatique.

Seules quatre espèces ont été observées, Cabomba aquatica, Ceratopteris thalictroides, Eichhornia heterosperma, en aval de Petit-Saut, et Eichhornia diversifolia sur le hautSinnamary.

\subsection{Les berges sableuses de rivière}

Les berges sableuses ont une grande importance floristique dans la dition. La strate arbustive est dominée par Licania leptostachya et Calliandra surinamensis. Les herbacées sont abondantes, avec Sauvagesia erecta, Cyperus millifolius, Rhynchospora cephalotes et Pariana campestris. Une seule fougère terrestre est présente: Thelypteris serrata.

\subsection{Les berges vaseuses de rivière}

La dynamique fluviale du Sinnamary ne permet la mise en place de berges boueuses que très en aval, au niveau de la zone influencée par les marées. Tout au plus trouve-t-on parfois quelques petits bancs de vases colonisés par Eleocharis interstincta.

\subsection{Les berges rocheuses de rivière}

Sur les rochers, peu d'espèces rupicoles si ce n'est Turnera rupestris très en aval (Hoff, 1993), une Cyperaceae: Hypolytrum longifolium et une fougère: Trichomanes hostmannianum.

\subsubsection{Les rochers des sauts}

Sur les sauts, nombreux dans tout le cours moyen du fleuve, plusieurs espèces sont relevées sur les rochers exondés: Henriettea succosa, Calliandra surinamensis, Rhynchospora reptans, Panicum laxum, Teliostachya cataractae. Sauvagesia erecta et $\mathrm{O}$ denlandia lancifolia dans les parties abritées. Mourera fluviatilis est très abondante dans le courant, surtout en 
amont des sauts. Plusieurs espèces d'Apinagia sont présentes, plutôt en aval du saut, dans les cascades, ainsi que Weddellina squamulosa. Les déterminations au niveau de l'espèce sont difficiles pour les Podostemoraceae. Très en amont, lorsque la hauteur d'eau ne dépasse pas quelques décimètres, d'autres Podostemonaceae entourent les cailloux du fond des rivières.

Comme l'indique Schnell (1965), l'écologie des divers peuplements de Podostemonaceae devrait être précisée.

\subsubsection{Les pripris}

Les vastes marécages herbacés et arbustifs, ou "pripris" de la zone littorale font place, plus au nord, dans les méandres des rivières à des formations plus arbustives qui sont souvent considérées comme des faciès des forêts marécageuses. Ces groupements sont dominés par Triplaris weigeltiana en strate arborescente, avec en bordure vers l'eau libre des rivières plusieurs Inga dont 1 . disticha, I. nobilis, I. pezizifera et surtout 1 . sertulifera. On trouve également Combretum rotundifolium et probablement Croton cuneatus. La strate basse est occupée par Montrichardia arborescens et Bonafousia siphilithica. Quelques arbustes lianescents sont présents, dont Allamanda cathartica. Les herbacees sont presque totalement absentes de ce groupement. Seules quelques Poaceae sont présentes, comme Panicum mertensi et Panicum laxum.

\subsubsection{Les groupements forestiers}

Trois forêts sont directement liées à l'eau. Elles se distinguent par l'écologie de leur substrat. Les forêts marécageuses sont toujours humides et l'eau y est presque toujours libre. Les forêts inondables, par contre, ont un substrat ferme et ce n'est que lors des grandes pluies de janvier-février ou de mai-juin que l'eau parcourt le sousbois. Le courant peut être assez élevé car le sous-bois est généralement bien nettoyé en fin de saison des pluies. Un apport de nutriment est alors également réalisé. Le substrat des forêts ripicoles, situées sur des talus abrupts, n'est que très rarement submergé et pendant de très courtes périodes avec des courants faibles.

\subsubsection{Les forêts marécageuses}

\subsection{Composition floristique}

La strate arborescente des forêts marécageuses est constituée par Symphonia globulifera, Caryocar microcarpum, Eschweilera pedicellata, Lecythis corrugata et Couratari gloriosa, avec Bertiera guianensis et Cordia nodosa dans les parties les plus fermes. Le sous bois est particulièrement riche en monocotylédones, avec Rapatea paludosa très abondant et Rapatea ulei, plusieurs Marantaceae, dont Calathea cyclophora, $C$. elliptica et $C$. propinqua, des Musaceae avec Heliconia lourteiggiae 
et $H$. spathocircinata, des Zingiberaceae comme Costus congestiflorus et des Arecaceae comme Geonoma baculifera.

\subsection{Ecologie}

Les forêts marécageuses présentent une hydromorphie permanente, avec la présence d'eau libre sous forme d'un réseau anastomosé. Lors des crues, les bases des ligneux sont submergées pendant des périodes plus ou moins longues. L'apport de nutriment lors des crues pourrait être positif car le sous-bois est assez dense, ce qui réduit fortement le courant et favorise les dépôts de matières organiques.

\subsubsection{Les forêts inondables}

\subsection{Composition floristique}

Les forêts inondables, ou forêts sur flats, sont dominées par Quararibea lasiocalyx et Heisteria acuminata. Le sous-bois est pauvre, avec peu d'espèces adaptées à des submersions plus ou moins longues, comme Psychotria racemosa, Leandra agrestis. Contrairement aux forêts marécageuses, les monocotylédones sont rares, si ce n'est une Arecaceae, Geonoma deversa et une Poaceae, Hypolytrum longifolium. Par contre, les Ptéridophytes semblent bien adaptées à ce milieu, avec Triplophyllum funestum, Lindsaea lancea, Lomogramma guianensis, Lomariopsis japurensis et Lygodium volubile (ces trois espèces étant lianescentes), Adiantum latifolium et Adiantum obliquum et, souvent très abondante, Selaginella parkeri.
Les épiphytes sont peu nombreuses. Or, la densité en épiphytes augmente avec l'âge du peuplement. Ceci indique que les forêts inondables ont un "turn-over" probablement nettement plus rapide que les autres types de forêts et que leur durée de vie ne permet pas l'installation de nombreuses espèces d'épiphytes.

\subsection{Ecologie et fonctionnement}

Les forêts inondables se caractérisent par un apport de nutriment lors des crues et par une érosion de la surface du sol (surtout par entraînement des feuilles mortes) lors de ces mêmes crues. Le bilan entre apport et érosion est probablement négatif. Le rapport s'inverse dès que le courant dépasse $1 \mathrm{~m} / \mathrm{s}$ (Cailleux, 1959). De plus, le sous-bois bas est peu dense (quelques fougères), ce qui ne ralentit que peu le courant et réduit donc les dépôts de matières organiques. D'après Cailleux, l'érosion des flats serait plus forte sur les berges des grandes rivières, à débit fort et continu, que sur les berges des petites rivières, à courant faible et discontinu.

Le sol des flats est constitué par un horizon limoneux à sable et gravier rares variant de 0 à 1 (2) $\mathrm{m}$ de profondeur et reposant sur des graviers. La nappe phréatique oscille à faible profondeur.

Le barrage créera de nouvelles forêts inondables, la crue étant remplacée par la hauteur d'eau du lac de barrage et par le marnage. Ces nouvelles forêts occuperont surtout des surfaces planes, topographie relativement rare dans la région. 


\subsubsection{Les forêts ripicoles}

\subsection{Composition floristique}

Les forêts ripicoles forment une frange de quelques mètres de largeur le long de la forêt dense de basse altitude. Elles sont, le plus souvent, situées sur les rives concaves, soumises à l'érosion. La flore y est très variée car elle associe des espèces de la forêt dense aux espèces de la voûte et aux héliophiles. La liste complète des espèces des forêts ripicoles de Guyane rassemble 1806 espèces appartiennent à cette formation, soit le $1 / 3$ de la flore connue.

Sur le Sinnamary, les arbres les plus communs sont des Caesalpiniaceae comme Eperua falcata et E. rubiginosa, Dicorynia guianensis, Macrolobium bifolium, Swartzia benthamiana et Vouacapoua americana. Les Chrysobalanaceae sont également bien représentées avec Hirtella bicornis, $H$. hispidula, Licania canescens, $L$. granvillei, L. leptostachya, $L$. licaniiflora, L. majuscula, L. membranacea et $L$. pallida. Paypayrola guianensis et Posoqueria latifolia sont particulièrement abondantes en sousbois ainsi que de nombreux Arecaceae: Geonoma baculifera, $G$. deversa et $G$. poiteauna.

Ces franges forestières sont également remarquables par la grande richesse en lianes comme la Fabaceae Dioclea $s p$. et diverses Malpighiaceae: Banisteriopsis lucida, Heteropterys acutifolia, $H$. macradena, $H$. nervosa. Les épiphytes sont également très nombreuses, avec des
Araceae : Philodendron grandifolium, $P$. squamiferum, des bromeliaceae: Pitcairnia leprieurii et Tillandsia monadelpha, des Orchidaceae: Psygmorchis pusilla, et de nombreuses fougères (Trichomanes crispum, $T$. elegans, Campyloneuron phyllitidis, $C$. repens, Cochlidium linearifolium, et Antrophyum guayanense.

Les herbacées sont un peu plus abondantes que dans les forêts inondables. Notons la grande Cyperaceae Mapania sylvatica, la Poaceae Ichnanthus pallens et la Zingiberaceae Costus congestiflorus. Les ptéridophytes sont abondantes, avec, en plus de celles citées en forêts inondables: Metaxya rostrata, Cyathea spectabilis, C. surinamensis et Selaginella falcata.

\subsection{Dynamique des forêts ripicoles}

D'après Cailleux (1959), la frange ripicole serait renouvelée tous les 40 ans, du moins pour la partie directement en contact avec le courant. Le turn-over est probablement plus lent sur la rive concave.

\subsection{Architecture des forêts ripicoles}

Une attention particulière doit être apportée à l'architecture des forêts. Les arbres présentent des variations morphologiques qui permettent la fermeture de la forêt vers la rivière. Oldeman (1972) présente plusieurs types de fermetures en fonction de l'architecture des arbres et de la topographie. On note deux grands types de fermeture: 
- fermeture par la modification de la direction de croissance moyenne des troncs des arbres. Celle-ci passe de la verticale à l'horizontale;

- fermeture par la modification dans la courbure des troncs. L'arbre est d'abord à croissance verticale, puis il se courbe vers la rivière.

Au bord de l'eau, par contre, certains axes se relèvent:

- les troncs à base horizontale dont l'extrémité se courbe et devient verticale;

- les rejets et les nouvelles unités de croissance sont d'emblée orthotropes.

Comment ces principes généraux se traduisent-ils dans la réalité ? Cela dépend de l'architecture des espèces. Nous ne reviendrons pas sur les modèles architecturaux (Hallé et al., 1970, Oldeman, 1974, Hallé et al., 1978), si ce n'est pour signaler trois observations de Oldeman :

- la stratification des branches s'intensifie du fait du comportement ripicole des espèces;

- des modèles orthotropes peuvent avoir, sous certaines conditions, des branches plagiotropes;

- les cimes ont un comportement asymétrique vers l'ouverture due à la rivière.

Ces éléments permettent de mieux comprendre l'architecture des rives. La courbe de croissance des troncs et l'asymétrie des cimes sont dues essentiellement à la lumière et à l'encombrement végétal. Ceci se traduit par un excès de croissance vers la rivière et donc par un déséquilibre de l'arbre par déplacement de son centre de gravité et une chute plus précoce qu'en forêt.

En conclusion, Oldeman souligne "qu'au bord des rivières, la voûte forestière descend jusqu'au niveau de l'eau". Cependant, la genèse, la structure et l'architecture de la frange ripicole est différente de celle de la voûte.

\subsubsection{Les pinotières}

\subsection{Composition floristique}

Les groupements à Euterpe oleracea, appelé "pinot" en Guyane, d'où le terme pinotière, sont peu individualisés et pauvres en espèces dans la zone considérée. Ils ne sont souvent qu'un faciès de la forêt marécageuse. Cependant, on trouve des pinotières perchées dans toutes les parties marécageuses de la forêt dense.

\subsection{Ecologie et fonctionnement}

Les pinotières se développent sur sol hydromorphe (Oldeman, 1969; Ricci, 1990) inondé en saison des pluies mais ressuyé en période sèche. Le substrat peut évoluer vers la pégasse, sol tourbeux riche en débris végétaux, à forte teneur en eau. II faut noter le système racinaire du palmier pinot avec des racines horizontales et des pneumatophores issus de ces racines.

\subsubsection{La forêt dense sempervirente} humide de basse altitude

La forêt dense sempervirente humide de basse altitude occupe tous les reliefs hors-eaux du bassin du Sinna- 
mary. Ce groupement, très complexe, a été étudié à proximité immédiate de la région étudiée, d'une part sur la crique Grégoire (Oldeman, 1974) et d'autre part sur la Piste de Saint-Elie (Sarrailh, 1990, Lescure et al. in Sarrailh 1990, Maury-Lechon in Sarrailh 1990, Sarrailh in Sarrailh 1990).

Nous ne reviendrons pas sur les nombreux travaux réalisés sur cette forêt tant sur son architecture et sa structure que sur son écologie et son fonctionnement. Nous renvoyons le lecteur aux travaux sus-cités qui présentent une importante bibliographie. Seul un rapide résumé est présenté ici.

Floristiquement (Lescure et al., 1990), les forêts denses de basse altitude de la Piste de Saint-Elie sont dominées par des Lecythidaceae (26\% des individus de plus de $20 \mathrm{~cm}$ de diamètre à $1,30 \mathrm{~m}$ ), des Caesalpiniaceae $(22 \%)$, des Chrysobalanaceae $(12 \%)$ et des Sapotaceae $(9 \%)$. Les trois premières familles sont aussi les plus riches en espèces: Caesalpiniaceae (24 especes), Lecythidaceae (20) et Chrysobalanaceae (16). Dans le sous-bois, les familles les plus fréquentes sont les Violaceae, Lecythidaceae, Annonaceae et Chrysobalanceae.

L'architecture de la forêt de la Crique Grégoire (Oldeman, 1974) se caractérise par plusieurs ensembles structuraux avec, en sous-bois un ensemble arbustif de 8 à $12 \mathrm{~m}$ de hauteur, puis un premier ensemble arborescent entre 18 et $22 \mathrm{~m}$ et un second entre 28 et $35 \mathrm{~m}$, constitué d'Eperua falcata et de Dicorynia guia- nensis. Enfin, quelques émergeants peuvent atteindre $45 \mathrm{~m}$. Cette forêt présente une assez grande richesse en lianes et en épiphytes.

Ecologiquement, on distingue classiquement quatre types de forêts en fonction du drainage (drainage vertical libre ou DVL et drainage vertical bloqué ou DVB) et de la présence ou l'absence d'hydromorphie de surface.

Les caractéristiques structurales et quantitatives de la forêt de la Piste de Saint-Elie sont les suivantes (Lescure et al., in Sarrailh, 1990) : la surface terrière varie de $18,5 \mathrm{~m}^{2} / \mathrm{ha}$ à $32,8 \mathrm{~m}^{2} / \mathrm{ha}$; la densité des ligneux de plus de $20 \mathrm{~cm}$ de diamètre varie de 142 à 286 individus par ha; la phytomasse est de l'ordre de 147,4 t/ha et la nécromasse de 22,7 t/ha; la production de litière est de $7,8 \mathrm{t} / \mathrm{ha} / \mathrm{an}$, celle de nécromasse de $11,7 \mathrm{t} / \mathrm{ha} / \mathrm{an}$; la productivité est de l'ordre de 12,3 tha/an.

\subsubsection{Superficie des groupements forestiers}

La superficie des différents types de forêts n'est pas connue avec précision. Sur les $6500 \mathrm{~km}^{2} \mathrm{du}$ bassin du Sinnamary, un peu plus de $300 \mathrm{~km}^{2}$ vont être submergés, soit environ $5 \%$ de la surface du bassin. D'après l'étude d'impact (EDF, 1985), la répartition des forêts qui seront inondées est la suivante:

Forêt de terre ferme sur roche verte : 16000 ha

Forêt de terre ferme sur autres roches: 7000 ha 
Ces deux types de forêts sont regroupés sous le terme de "forêt dense sempervirente humide de basse altitude".

Forêt de bas-fond hydromorphe: 7000 ha

La forêt de bas-fond hydromorphe regroupe les forêts marécageuses, les forêts inondables, les pripris et les pinotières et les forêts ripicoles.

\section{Total : 30000 ha}

Les forêts ripicoles au sens large donc directement liées au cours du fleuve et de ses affluents, représentent environ $23 \%$ du total de la surface forestière inondée. Ces chiffres pourraient être précisés par l'utilisation des images satellitaires.

\subsection{Répartition spatiales des groupements de berges}

La connaissance des groupements de berges du Sinnamary actuel est déterminante pour étudier les variations et les évolutions qui vont résulter de la mise en eau du barrage. Les $470 \mathrm{~km}$ de berges actuelles du Sinnamary et des ses affluents principaux inclus dans la future zone inondée $(235 \times 2)$ vont disparaître et seront remplacées par $1050 \mathrm{~km}$ de "nouvelles berges", limitant le plan d'eau de la retenue (Sissakian, 1992).

Afin de suivre cette évolution, 140 $\mathrm{km}$ de berges $(2 \times 76,5 \mathrm{~km})$ entre Saut Vata et Saut Stéphanie, ont été cartographiée, sur les $470 \mathrm{~km}$. La zone a été choisie pour deux raisons. D'une part elle a été très peu modifiée par l'homme. En aval de Saut Vata, et jusqu'à Petit-Saut, les forêts secondarisées sont, par contre, importantes (bagne de Annamites de Saut Tigre, prospection aurifère). D'autre part, elle inclue une zone qui ne sera pas inondée, en amont de Saut Takari-Tanté jusqu'à Saut Stéphanie. Cette zone servira de témoins pour analyser les modifications qui interviendront en aval.

Quatre groupements végétaux principaux ont été cartographié entre Saut Vata et Saut Stéphanie :

- la forêt inondable, ou forêt sur flat (Carte 2);

- la frange ripicole de la forêt dense de basse altitude (Carte 3 );

- la forêt marécageuse (Carte 4);

- le pripris (Carte 5).

Le tableau 2 présente les longueurs des différents groupements de berges le long du Sinnamary entre Saut Vata et Saut Stéphanie.

Le cours du Sinnamary se divise en plusieurs sections ayant chacune une répartition particulière des groupements végétaux de berge, et donc un fonctionnement écologique propre. Pour des raisons de reliefs, les sections sont limitées généralement par des sauts. Au total, les forêts inondables sur flats occupent $50 \%$ des berges, suivies par les forêts marécageuses $(20 \%)$. Les pripris et les forêts ripicoles ne couvrent que $14 \%$ des berges. Les forêts inondables sont réparties presque uniformément. Par contre, les pripris et les forêts marécageuses sont nettement plus importantes dans le cours supérieur, après Saut Takari-Tanté, tandis que les fo- 

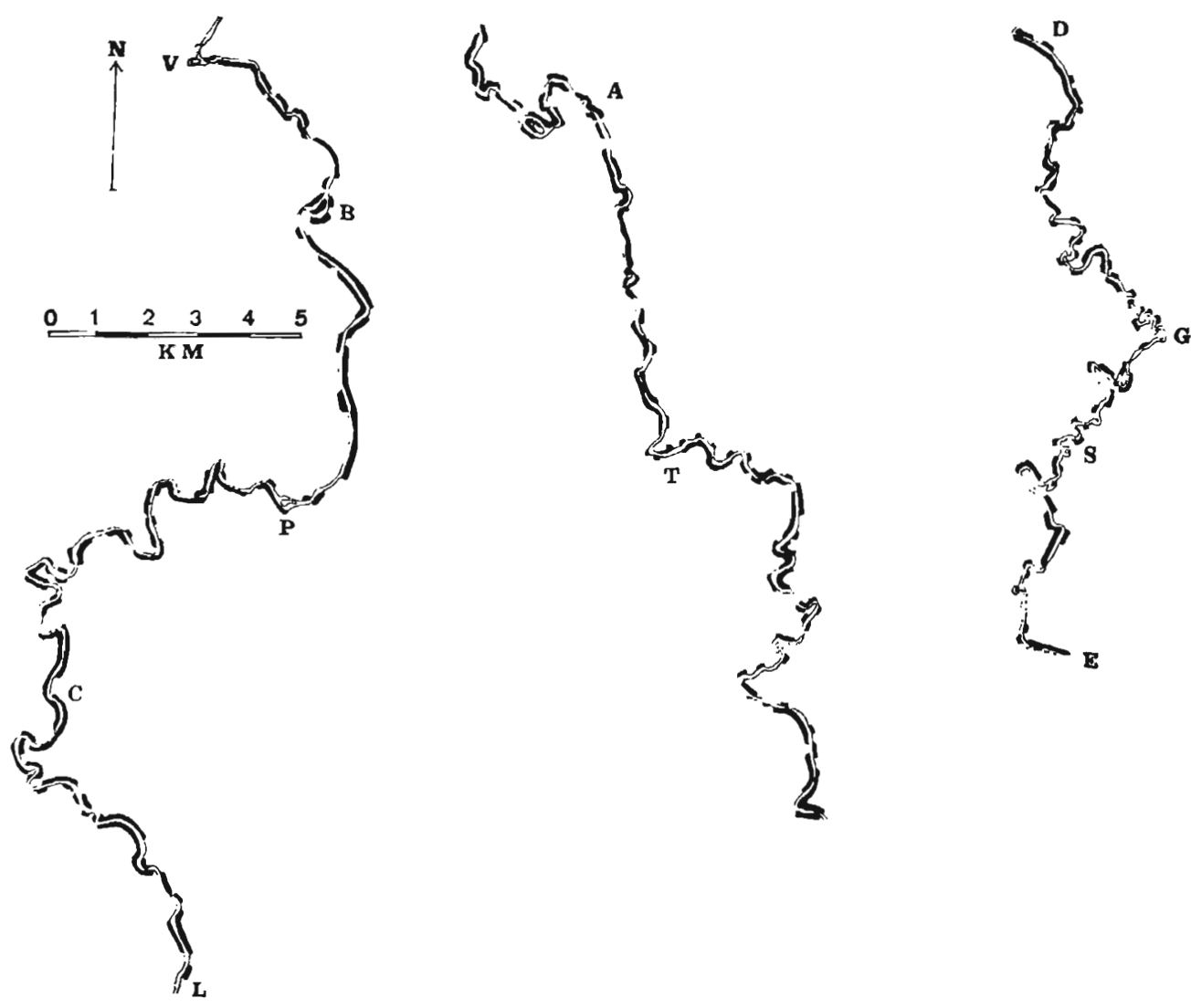

Carte 2. - Carte des forêts inondables entre Saut Vata et Saut Stéphanie.

En trait épais: présence du groupement

V: Saut Vata, B: Saut Bérard, P: Saut Patawa, C: Latitude $4^{\circ} 45^{\prime}$, L: Saut L'Autel, A: Saut Aïmara, T : Saut Takari-Tanté. D : Saut Dalles, G: Latitude 4030', S: Saut Stéphanie, E : Fin Map 2. - Map of flooded forest between Saut Vata and Saut Stéphanie.

Thick line : presence of the vegetation

V: Saut Vata, B: Saut Bérard, P: Saut Patawa, C: Latitude $4^{\circ} 45^{\circ}$, L: Saut L'Autel, A : Saut Aïmara, T : Saut Takari-Tanté. D : Saut Dalles, G: Latitude 4030', S : Saut Stéphanie, E : Fin 

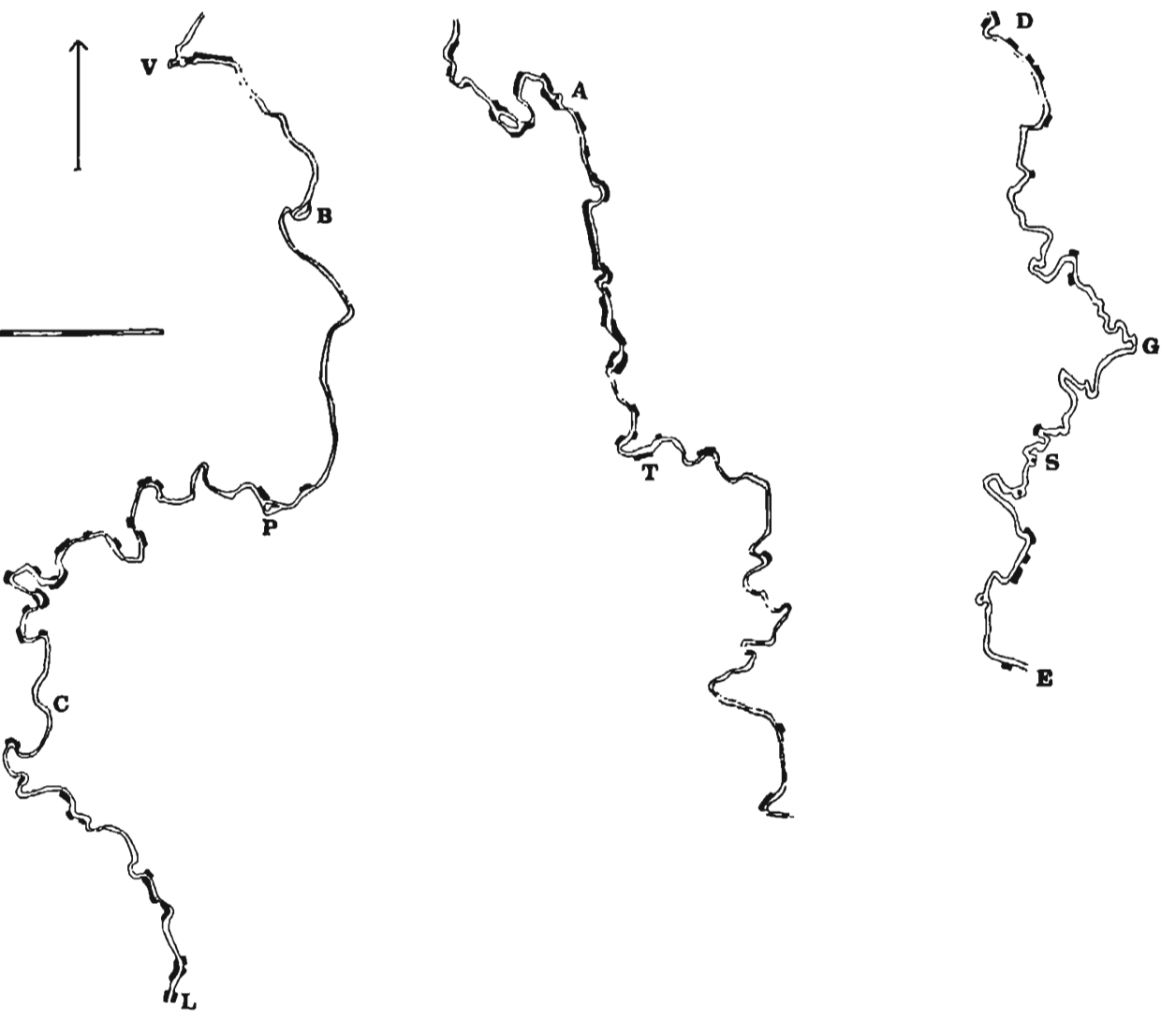

Carte 3. - Carte des forêts ripicoles entre Saut Vata et Saut Stéphanie.

En trait épais : présence du groupement

V: Saut Vata, B : Saut Bérard, P: Saut Patawa, C : Latitude 4045', L: Saut L'Autel, A : Saut Aïmara, T : Saut Takari-Tanté, D : Saut Dalles, G : Latitude 4030', S : Saut Stéphanie,E : Fin

Map 3. - Map of ripicolous forest betwenn Saut Vata et Saut Stephanie

Thick line : presence of the vegetation

V: Saut Vata, B: Saut Bérard, P: Saut Patawa, C : Latitude 4045', L: Saut L'Autel, A: Saut Aïmara, T : Saut Takari-Tanté, D : Saut Dalles, G: Latitude 4030', S : Saut Stéphanie, E : Fin 


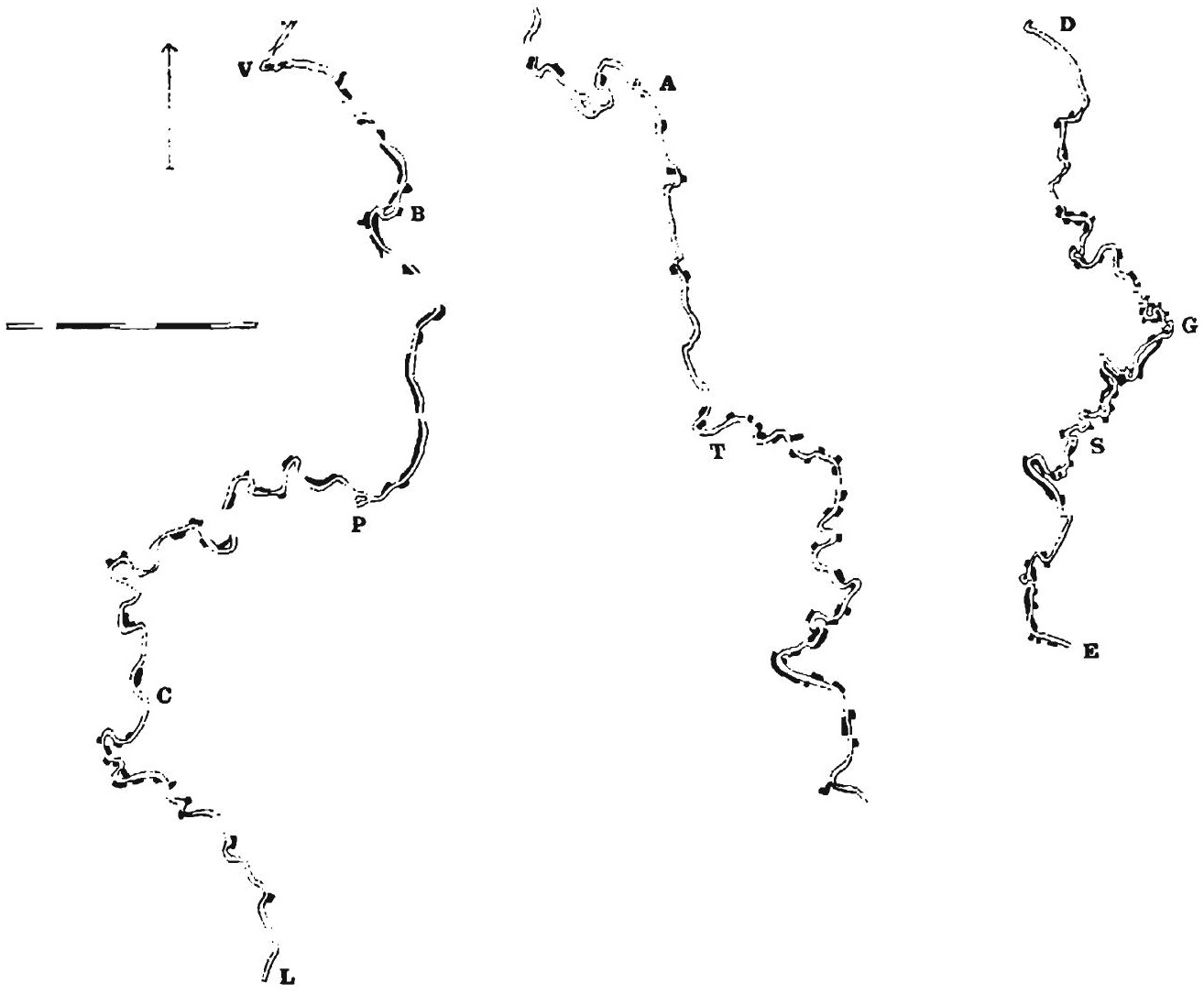

Carte 4. - Carte des forêts marécageuses entre Saut Vata et Saut Stéphanie.

En trait épais: présence du groupement

V: Saut Vata, B: Saut Bérard, P: Saut Patawa, C: Latitude 4045', L: Saut L'Autel, A: Saut Aïmara, T: Saut Takari-Tanté, D : Saut Dalles, G: Latitude 4³0', S: Saut Stéphanie, E : Fin Map 4. - Map of swamp forest between Saut Vata et Saut Stéphanie.

Thick line: presence of the vegetation

V: Saut Vata, B: Saut Bérard, P: Saut Patawa, C: Latitude $4^{\circ} 45^{\circ}$, L: Saut L'Autel, A : Saut Aïmara, T: Saut Takari-Tanté, D : Saut Dalles, G: Latitude 4030', S : Saut Stéphanie, E : Fin 

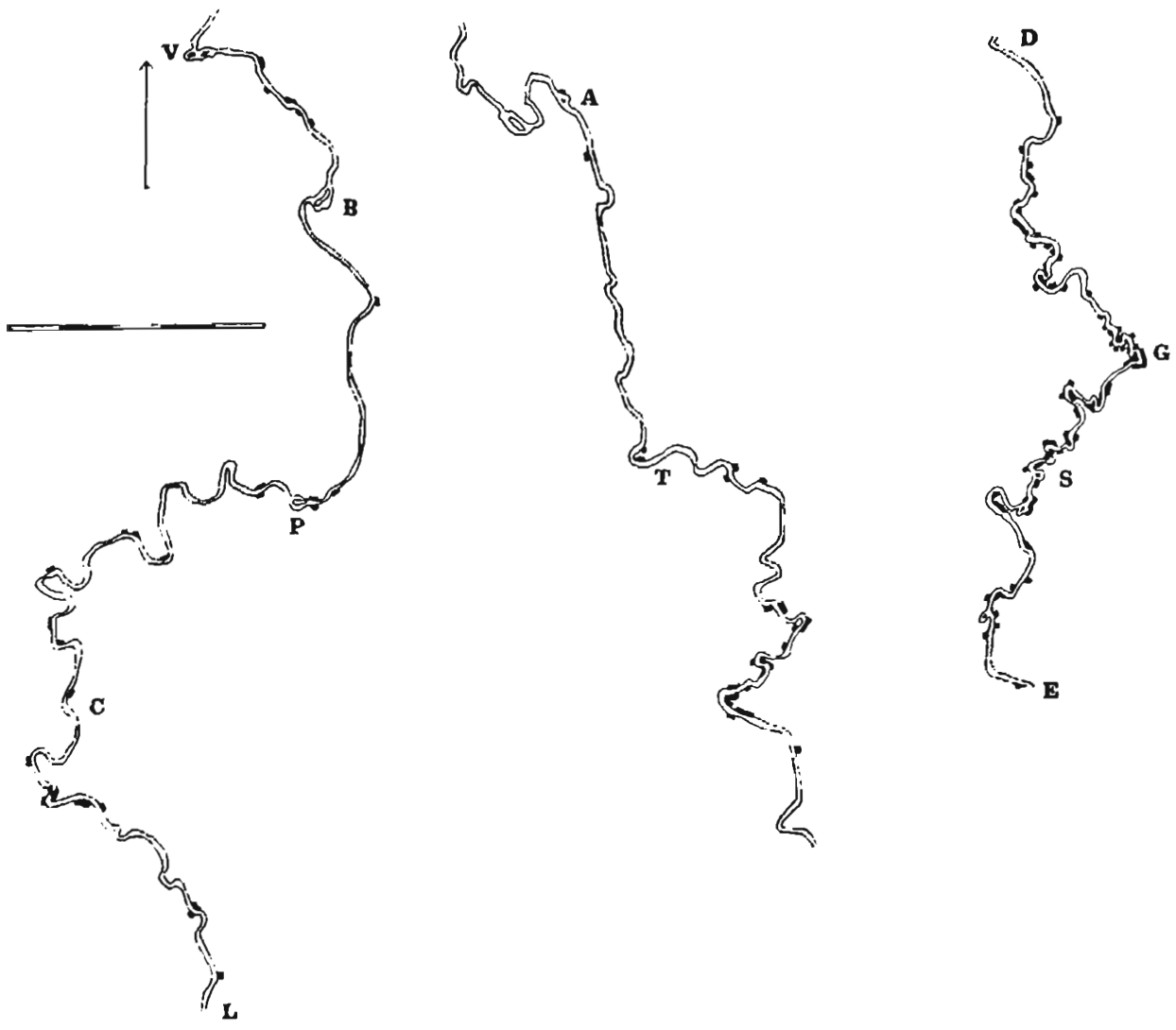

Carte 5. - Carte des pripris entre Saut Vata et Saut Stéphanie.

En trait épais : présence du groupement

V: Saut Vata, B: Saut Bérard, P: Saut Patawa, C: Latitude 4045', L: Saut L’Autel, A: Saut Aimara, T: Saut Takari-Tanté, D: Saut Dalles, G: Latitude 4030', S: Saut Stéphanie, E : Fin Map 5. - Map of swamp shrub "pripris" between Saut Vata et Saut Stéphanie.

Thick line: presence of the vegetation

V: Saut Vata, B: Saut Bérard, P: Saut Patawa, C: Latitude 4045', L: Saut L'Autel, A : Saut Aïmara, T : Saut Takari-Tanté, D : Saut Dalles, G : Latitude 4030', S : Saut Stéphanie, E : Fin 
Tableau 2. - Longueur des groupements végétaux le long du Sinnamary entre Saut Vata et Saut Stéphanie

Table 2. - Length of types of vegetation along the Sinnamary between Saut Vata and Saut Stéphanie

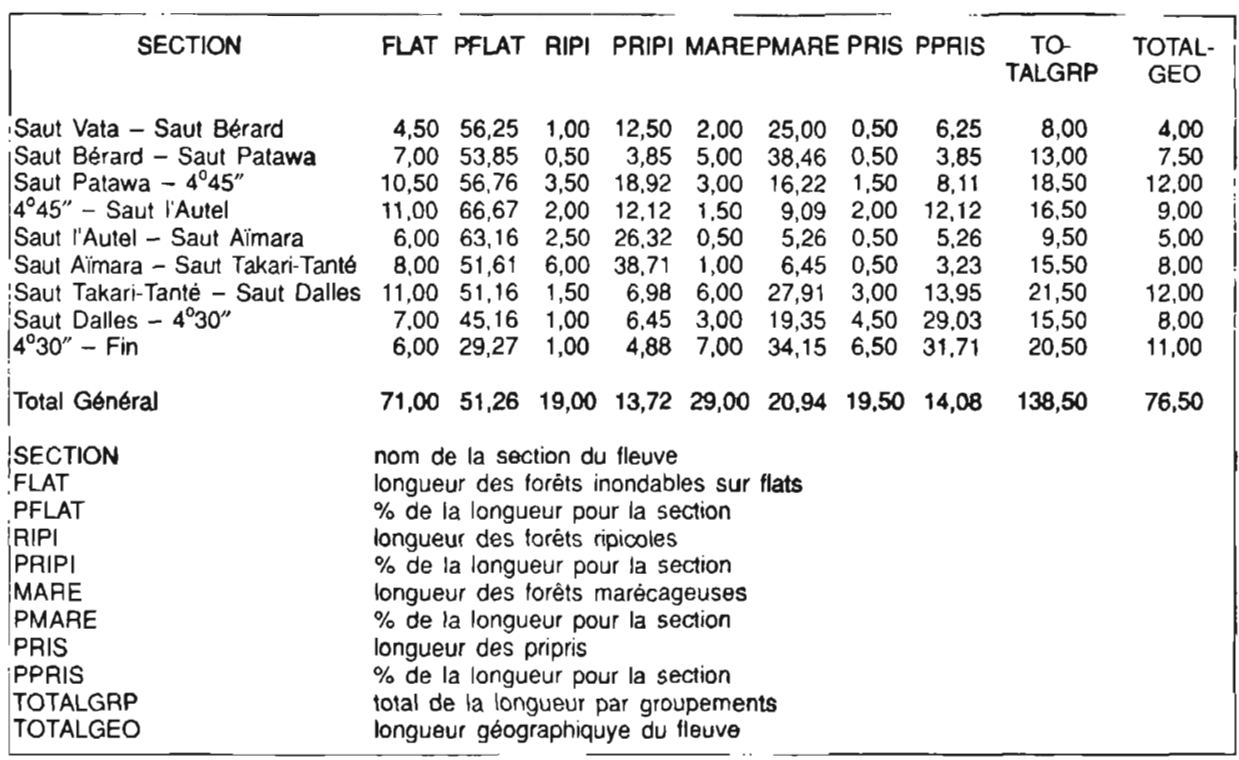

rêts ripicoles sur talus dominent dans le cours moyen, entre Saut l'Autel et saut Takari-Tanté.

La végétation des berges du Sinnamary se présente de la manière suivante :

- de Saut Vata à Saut Patawa: les forêts inondables et les forêts marécageuses dominent;

- de Saut Patawa à Saut TakariTanté : les forêts ripicoles proprement dites sont importantes, peu de forêts marécageuses ou de pripris;

- de Saut Takari-Tanté à $4^{\circ} 30^{\prime}$ : importance des forêts marécageuses et des forêts inondables, comme dans la première section;
- au sud de $4^{\circ} 30^{\prime}$ : développement des pripris et des forêts marécageuses.

Les berges du fleuve ne sont pas uniformes, le lac de retenue, qui n'atteindra, au maximum, qu'une profondeur de $35 \mathrm{~m}$ près de Petit-Saut, ne pourra pas être homogène, ni sur le plan physique, ni sur le plan chimique, comme le note C. Sissakian (1992). A l'aide de la télédétection, une partition du cours du Sinnamary et de ses affluents, sur une base phytoécologique, pourrait être envisagée, puis mise en relation avec les caractéristiques des différentes zones proposées par C. Sissakian (o.c.). 


\section{CONCLUSION}

L'étude d'environnement du site du barrage de Petit-Saut a permis de décrire une dizaine de formations végétales le long des $140 \mathrm{~km}$ de berges du Sinnamary entre Saut Vata et Saut Stéphanie: groupements végétaux ouverts (berges sableuses et rocheuses, pripris) ou fermés (forêts marécageuses, inondables et ripicoles). La répartition de ces groupements végétaux le long du Sinnamary n'est pas uniforme. La partition du fleuve en plusieurs zones en fonction de la distribution des formations végétales est possible. Elle pourra être confirmée à partir des images satellitaires.

Les sauts ont constitué un attrait paysager majeur du fleuve. La plupart sont désormais recouverts par les eaux du lac du barrage. La description et la cartographie de quelques sauts et rapides ont permis de mieux préciser leur géomorphologie.

Le barrage va créer une retenue d'environ $300 \mathrm{~km}^{2}$, soit $5 \%$ du bassin versant du Sinnamary (environ $6000 \mathrm{~km}^{2}$ ). Les groupements végétaux riverains seront fortement modifiés par ce barrage, non seulement du fait de l'inondation, mais aussi par la création de plus de $1050 \mathrm{~km}$ de nouvelles berges, au lieu des $470 \mathrm{~km}$ de berges actuelles.

\section{REMERCIEMENTS}

Les travaux présentés dans ce rapport sont les fruits de plusieurs missions sur le Sinnamary. Je tiens à remercier tous mes camarades de terrain qui ont organi- sé ou participé à certaines de ces missions, et plus particulièrement l'équipe d'hydrobiologie du Centre ORSTOM de Cayenne, avec L. Tito de Morais et L. Lauzanne, le laboratoire d'environnement de Petit-Saut avec P. Cerdan, S. Richard et V. Horeau, ainsi que nos piroguiers $G$. Elfort, A. Amesina, P. Villanove. Mes collègues G. Cremers et J.J. de Granville ont bien voulu relire cet article pour y apporter leurs compléments. B. Bordenave a cartographié certains sauts. Les travaux ont été financé par une convention ORSTOMEDF.

\section{AUTEURS CITÉS}

Bonnet M., Vaquer A., Champeau A., Grégoire A., 1988. Etude des macrophytes aquatiques de Guyane dans le cadre du projet de barrage de Petit-Saut. Rapport final du contrat E 3443 (SEI - ADER) : 1-36.

Boyé M. et al., 1978. Atlas des Départements Français d'Outre-Mer. IV. La Guyane. CNRS - ORSTOM.

Boyé M., Cabaussel G., Perrot Y., 1978. Climatologie. In: Atlas des départements d'outre-mer, 4. La Guyane; 1979. Edition CNRS-ORSTOM, Bordeaux-Talence.

Cailleux A., 1959. Etude sur l'érosion et la sédimentation en Guyane. In: Choubert, B., 1959. Essai sur la morphologie de la Guyane. Mémoires pour servir à l'explication de la carte géologique de la France: Département de la Guyane. Conférence géologique des Guyanes, septembre 1957. 1-48.

Cerdan P., Horeau V., Richard S., 1993. Les Sphingidae de forêt en Guyane française: Comparaison des populations en forêt inondable et non inondable. Hydroécol. Appl, 5, (2): 97-106.

Champeau A., 1991. L'hydrobiologie du site de Petit-Saut. Aménagement Hydro-Electrique de Petit-Saut (Guyane). Compte-rendu des pre- 
mières journées d'environnement relatives à la protection du site de PetitSaut. Cayenne 25-27 mars 1991. Multigr. EDF : 57-63.

Champeau A., Vaquer, A., Grégoire, A., 1991. Petit-Saut hydroelectric scheme : vegetal associations of stagnant waters in French Guyana. Hydroécol. Appl., 3, (1) : 111-124.

Choubert B., 1959. Essai sur la morphologie de la Guyane. Mémoires pour servir à l'explication de la carte géologique de la France: Département de la Guyane. Conférence géologique des Guyanes, septembre 1957. 1-48.

Cremers G., Hoff. M., 1991a. Etude d'environnement et d'impact concernant la route Régina - Saint-Georges. Guyane. Botanique et écologie végétale. Rapport définitif. Convention ORSTOM - DDE Multigr. Centre ORSTOM de Cayenne. 62 p.

Dessillons O., Vaquer A., Champeau A., Grégoire A., 1989. Etude des macrophytes aquatiques de Guyane dans le cadre du projet de barrage de PetitSaut. Rapport final du contrat SE 3592 (SEI - Cabinet Bressac) : 1-282.

Donselaar J. van, 1989. The vegetation in the Brokopondo Lake basin (Surinam) before, during, and after the inundation, 1964-1972. Brokopondo Research Report, Suriname, part III, Nat. Wet. Studiekr. Sur. Ned. Ant., Amsterdam, 124: 1-45.

Electricité de France, 1985. Chute de Petit Saut. Aménagement du Sinnamary. Demande de concession et demande de déclaration d'utilité publique. Dossier d'Enquète. EDF, Centre de Guyane. 1-503.

Granville J.-J. de, 1978. Planche Végétation. In: Atlas des départements d'outre-mer, 4. La Guyane. Edition CNRS-ORSTOM, Bordeaux-Talence.

Granville J.-J. de, 1986. Les formations végétales de la bande côtière de Guyane Française. In : Le littoral guyanais. Fragilité de l'environnement. $\mathrm{Na}$ - ture Guyanaise, Numéro Hors Série; $X^{\circ}$ colloque SEPANRIT, 1er Congrès Régional de l'Environnement, Cayenne. 47-63.

Granville J.-J. de, 1992. Les formations actuelles des zones côtières et subcôtières des Guyanes. In: Evolution des littoraux de Guyane et de la zone caraibe méridionale pendant le quaternaire. Coll. Colloque et Séminaires, ORSTOM. 231-250.

Hallé F. et Oldeman R.A.A., 1970. Essai sur l'architecture et la croissance des arbres tropicaux. Masson, Paris, 1 164.

Hallé F., Oldeman R.A.A. \& Tomlinson P.B., 1978. Tropical trees and forests : an architectural analysis. Springer. Berlin.

Hiez G., et Dubreuil P., 1964. Les régimes hydrologiques en Guyane française. Mém. ORSTOM, $3:$ 1-119.

Hoff M., Brisse H., 1985. Mise en place d'une base de données en botanique tropicale. 1. Informatisation des échantillons d'herbier. Coll. Information - Documentation Technique, ORSTOM, Paris, $65: 1-44$.

Hoff M., Cremers G., Feuillet C., Granville J.J. de, 1989. La banque de Données "AUBLET" de l'Herbier du Centre ORSTOM de Cayenne (CAY). Bull. Jard. Bot. Nat. Belg., 59 : 171-178.

Hoff M., Brisse H., 1990. Diversité et répartition des formations végétales en Guyane française à partir d'une banque de données sur l'environnement. Atelier sur l'Aménagement et la Conservation de l'Ecosystème forestier tropical humide. Cayenne 12-16 mars $1990: 1-25$.

Hoff M., 1991, Liste des Milieux, Habitats et Formations végétales de Guyane. Collection Patrimoines Naturels, Vol. 5, Série Patrimoine Ecologique. Secrétariat de la Faune et de la Flore, Muséum national d'Histoire naturelle, Paris. 1-24. 
Hoff M., 1993. Contribution à l'étude des Turneraceae des Guyanes: Turnera rupestris Aublet, espèce menacée de disparition en Guyane. Acta Botanica Gallica, 140, (3) : 291-299.

Hoff M., Reynaud, P.A., Toriola-Marbot, D., Deshayes, C., 1995. Un marais tropical : Le Grand Pripris de Yiyi (Guyane française). Bilan écologique du site naturel protégé de la Crique Yiyi. Rapport final de la Convention ORSTOM Conservatoire du Littoral. : 1-114.

Hoff M., 1993. Un fleuve tropical, le Sinnamary. Etude de la Flore et de la Végétation du Site du barrage de Petit-Saut et de ses environs. Rapport final de la Convention ORSTOM EDF : 1-388.

Hoff M., 1994. Biodiversité floristique d'un bassin fluvial tropical: Le Sinnamary (Guyane française). Ecologie, 25, (3) : 189-200.

Lamotte S., 1992. Essai d'Interprétation Dynamique des Végétations en Milieu Tropical Inondable. La Plaine Alluviale de Haute-Amazonie. Thèse, Université de Montpellier II, 1-423.

Lauzanne L., Tito de Morais L., Tito de Morais A., Ponton D., 1993. Structure et biologie des peuplements ichtyques du fleuve Sinnamary en Guyane française. Rapport final, ORSTOM - EDF, 1-133 + annexes.

Léonard J., 1993. Etude phytosociologique des chutes de la Tshopo (Kisangani - Zaïre). Bull. Jard. Bot. Nat. Belg., 62 : 283-347.

Lescure J.P., Puig H., Riéra B., Sabatier D., 1990. Une forêt primaire de Guyane française: données botanique. In: Sarrailh, J.M., 1990 - Mise en valeur de l'écosystème forestier guyanais. Opération Ecerex. INRA CTFT, Coll. Ecologie et Aménagement Rural. 137-168.

Lindeman J.C., 1953. The vegetation of the coastal region of Suriname. Utrecht, Keminke en zoon N.V. 1-135.
Maury-Lechon G., 1990. La régénération. A. En forêt naturelle témoin. In: Sarrailh, J.M., 1990 - Mise en valeur de l'écosystème forestier guyanais. Opération Ecerex. INRA - CTFT, Coll. Ecologie et Aménagement Rural. 169-185.

Oldeman R.A.A., 1969. Etude biologique des pinotières de la Guyane française. Cahiers ORSTOM, Série Biol., 10 : 318.

Oldeman R.A.A., 1972. L'architecture de la végétation ripicole forestière des fleuves et criques guyanais. Adansonia. N.S., 12, (2) : 253-265.

Oldeman R.A.A., 1974. L'architecture de la forêt guyanaise. Mémoires ORSTOM $n^{\circ} 73$. Paris. 1-204.

Ricci J.P., 1990. Les pinotières. Bois et Forêts des tropiques. Spécial Guyane. $\mathrm{N}^{\circ} 220: 55-63$.

Sarrailh J.M., 1990. Mise en valeur de l'écosystème forestier guyanais. Opération Ecerex. INRA - CTFT, Coll. Ecologie et Aménagement Rural. 1-273.

Sarrailh J.M., Foresta H. de, Maury-Lechon G., Prévost M.F., 1990. La régénération. A. Après coupe papetière: parcelle Arbocel. In: Sarrailh, J.M., 1990 - Mise en valeur de l'écosystème forestier guyanais. Opération Ecerex. INRA - CTFT, Coll. Ecologie et Aménagement Rural. 187-208.

Schnell R., 1965. Aperçu préliminaire sur la phytogéographie de la Guyane. Adansonia, n.s., 5, (3) : 309-355.

Sissakian C., 1992. Présentation de la retenue de Petit-Saut en Guyane française: cartographie-partition de la retenue - volumes et surfaces - intégration paysagère. Hydroécol. Appl., 4, (1) : 121-132.

Traore D., Anoma G., Egnankou W., 1990. Végétation et flore de milieux hydrophytiques ouverts: barrages et basfonds de rivières, du Centre-Est de la Côte d'lvoire. Candollea, 45 : 423-438.

Werkhoven M.C.M., Peeters G.M.T., 1993. Aquatica macrophytes. In : Paul E. Ouboter (ed.), Freshwater Ecosystems of Suriname, : 99-112. 


\section{Annexe 1}

\section{Liste des espèces non forestières des berges du Sinnamary}

\section{DICOTYLEDONES}

ACANTHACEAE

Ruellia rubra Aublet

Teliostachya cataractae Nees

\section{ANNONACEAE}

Froesiodendron surinamense (R.E. Fries)

R.E. Fries

Guatteria guianensis (Aublet) R.E. Fries

\section{APOCYNACEAE}

Allamanda cathartica Linnaeus

Bonafousia siphilitica (Linnaeus f.) Allorge

Malouetia tamaquarina (Aublet) A. De

Candolle

var. minor De Candolle

Mesechites trifida (N.J. Jacquin) MuellerArgoviensis

\section{ASTERACEAE}

Rolandra fruticosa (Linnaeus) O. Kuntze Sparganophorus sparganophora (Linnaeus) C. Jeffrey

\section{BOMBACACEAE}

Quararibea guianensis Aublet

\section{CABOMBACACEA}

Cabomba aquatica Aublet

\section{CAESALPINIACEAE}

Eperua rubiginosa Miquel

Peltogyne venosa (Vahl) Bentham

Senna multijuga (L.C. Richard) Irwin \& Barneby

Senna quinquangulata (L.C. Richard) Irwin \& Barneby

\section{CAPPARACEAE}

Cleome aculeata Linnaeus

\section{CHRYSOBALANACEAE}

Licania incana Aublet

Licania leptostachya Bentham

\section{COMBRETACEAE}

Combretum rotundifolium L.C. Richard

\section{DILLENIACEAE}

Doliocarpus major J.F. Gmelin

\section{EUPHORBIACEAE}

Conceveiba guianensis Aublet

Croton cuneatus Klotzsch

\section{FLACOURTIACEAE}

Casearia pitumba Sleumer

Ryania speciosa Vahl

var. tomentosa (Miquel) Monachino

\section{GENTIANACEAE}

Coutoubea ramosa Aublet

Irlbachia alata (Aublet) Maas

\section{LAMIACEAE}

Hyptis mutabilis (Richard) Briquet

\section{LECYTHIDACEAE}

Eschweilera pedicellata (Richard) Mori

Gustavia augusta Linnaeus

Lecythis idatimon Aublet

\section{MARCGRAVIACEAE}

Marcgravia coriacea Vahl

\section{MELASTOMATACEAE}

Henriettea succosa (Aublet) De Candolle Macrocentrum cristatum (De Candolle) Triana

var. cristatum

Nepsera aquatica (Aublet) Naudin

\section{MELIACEAE}

Guarea gomma Pulle

\section{MIMOSACEAE}

Calliandra surinamensis Bentham

Inga disticha Bentham

Inga nobilis Willdenow

Inga pezizifera Bentham

Inga sertulifera De Candolle

\section{OCHNACEAE}

Ouratea leblondii (Van Tieghem) Lemée

Sauvagesia erecta Linnaeus

\section{ONAGRACEAE}

Ludwigia decurrens Walter

Ludwigia erecta (Linnaeus) Hara

Ludwigia hyssopifolia (G. Don) Exell

\section{PASSIFLORACEAE}

Passiflora citrifolia (Jussieu) Masters

\section{PIPERACEAE}

Peperomia macrostachya (Vahl) A. Dietrich

\section{PODOSTEMACEAE}

Mourera fluviatilis Aublet

Weddellina squamulosa Tulasne

\section{POLYGONACEAE}

Triplaris weigeltiana (Reichenbach) 0.

Kuntze

\section{RHIZOPHORACEAE}

Rhizophora racemosa G.F.W. Meyer

RUBIACEAE

Borreria latifolia (Aublet) K. Schumann 
Manettia alba (Aublet) Wernham

Oldenlandia lancifolia (K. Schumann) De

Candolle

Posoqueria latifolia (Rudge) Roemer \&

Schultes

Psychotria hoffmannseggiana (Roem. \&

Schult.) M.-Arg.

Psychotria moroidea Steyermark

\section{SAPINDACEAE}

Pseudima frutescens (Aublet) Radlkofer

Serjania grandifolia Sagot

\section{TURNERACEAE}

Turnera rupestris Aublet

var. rupestris

\section{VERBENACEAE}

Aegiphila integrifolia (N.J. Jacquin) B.D. Jackson

\section{MONOCOTYLEDONES}

\section{ARACEAE}

Anthurium gracile (Rudge) Lindley

Montrichardia arborescens (Linnaeus)

Schott

\section{ARECACEAE}

Desmoncus macroacanthos Martius

\section{BROMELIACEAE}

Guzmania lingulata (Linnaeus) $\mathrm{Mez}$

Tillandsia bulbosa Hooker

\section{CYPERACEAE}

Calyptrocarya glomerulata (Brongniart) Urban

Cyperus luzulae (Linnaeus) Retzius

Cyperus miliifolius Poeppig \& Kunth

Eleocharis interstincta (Vahl) Roemer ot

Schultes

Hypolytrum tongifolium (L.C. Richard) Nees

subsp. sylvaticum (Poeppig \& Kunth) T. Koyama

Rhynchospora cephalotes (Linnaeus) Vahl Rhynchospora pubera (Vahi) Böckeler Rhynchospora reptans (L.C. Richard) Kükenthal

Scleria secans (Linnaeus) Urban

\section{ERIOCAULACEAE}

Tonina fluviatilis Aublet

\section{LILIACEAE}

Hymenocallis tubiflora Salisbury

\section{MAYACACEAE}

Mayaca fluviatilis Aublet

ORCHIDACEAE

Ionopsis utricularioides (Swartz) Lindley

Octomeria petulans Reichenbach $\mathrm{f}$.

POACEAE

Olyra longifolia Kunth in Humboldt, Bonpland \& Kunth

Olyra obliquifolia Steudel

Panicum laxum Swartz

Panicum mertensii Roth in Roemer \&

Schultes

Panicum pilosum Swartz

Pariana campestris Aublet

\section{PONTEDERIACEAE}

Eichhornia diversifolia (Vahl) Urban

Eichhornia heterosperma Alexander

RAPATEACEAE

Rapatea paludosa Aublet

THURNIACEAE

Thurnia sphaerocephala (Rudge) J.D.

Hooker

\section{PTERIDOPHYTES}

DENNSTAEDTIACEAE

Lindsaea dubia Sprengel

Lindsaea reniformis Dryander

\section{GLEICHENIACEAE}

Dicranopteris pectinata (Willdenow) Underwood

\section{HYMENOPHYLLACEAE}

Hymenophyllum myriocarpum Hooker

Trichomanes crispum Linnaeus

Trichomanes cristatum Kaulfuss

Trichomanes hostmannianum (Klotzsch) Kunze

\section{POLYPODIACEAE}

Microgramma reptans (Cavanilles) A.R. Smith

\section{PTERIDACEAE}

Ceratopteris thalictroides (Linnaeus) Brongniant

\section{THELYPTERIDACEAE}

Thelypteris serrata (Cavanilles) Alston 


\section{Annexe 2}

\section{Cartes détaillées des 22 sauts et berges remarquables}
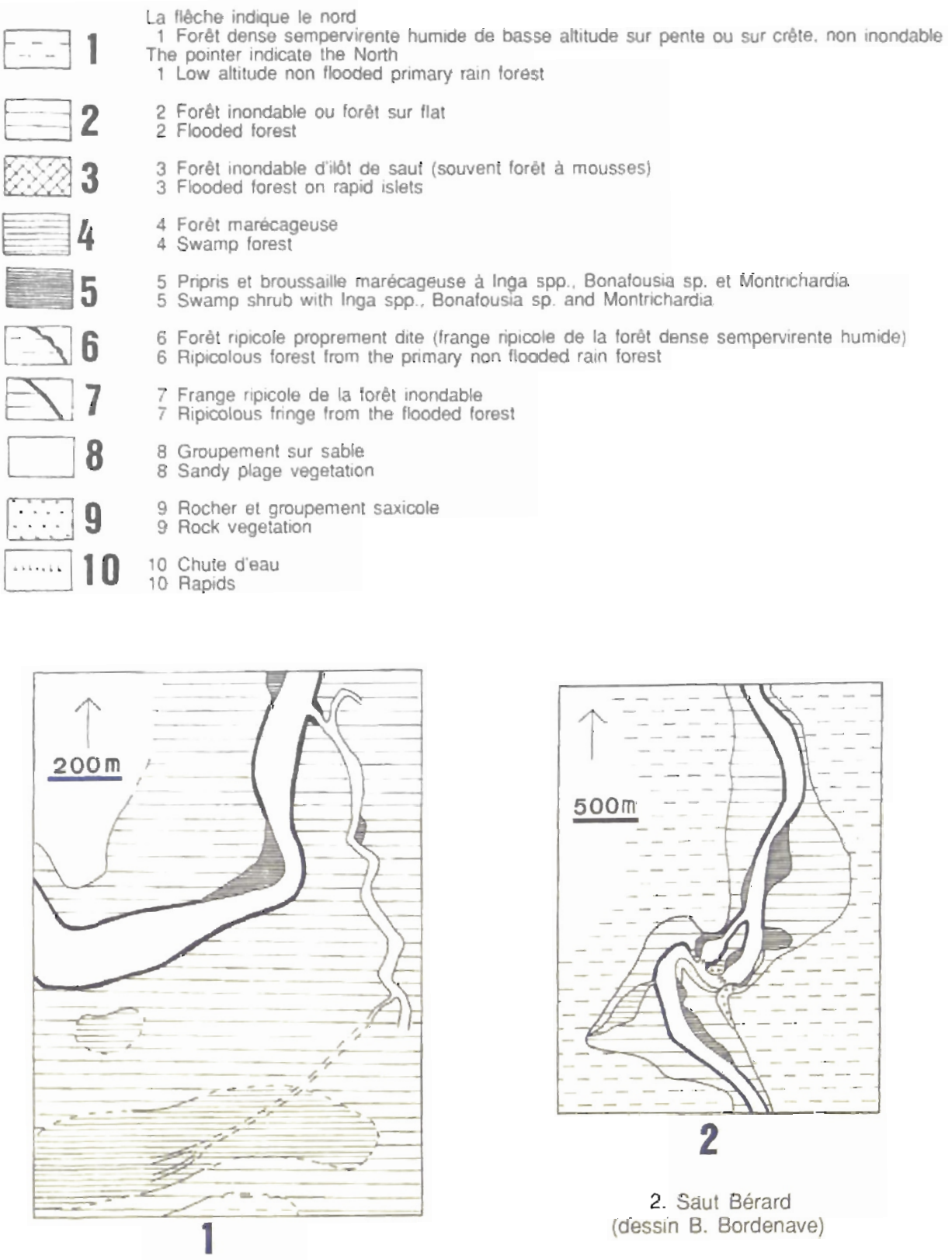

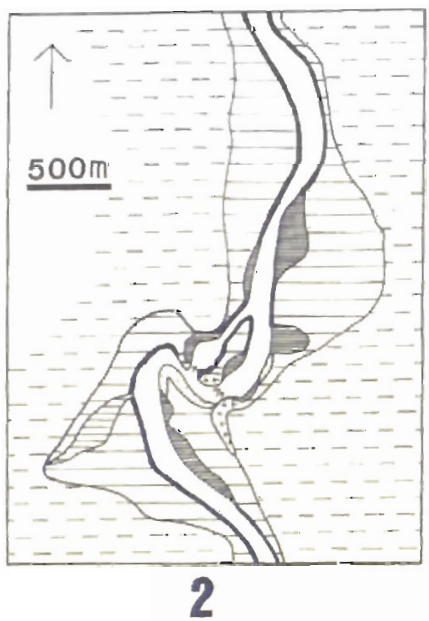

2. Saut Bérard (dessin B. Bordenave)

1. Crique Vénus 


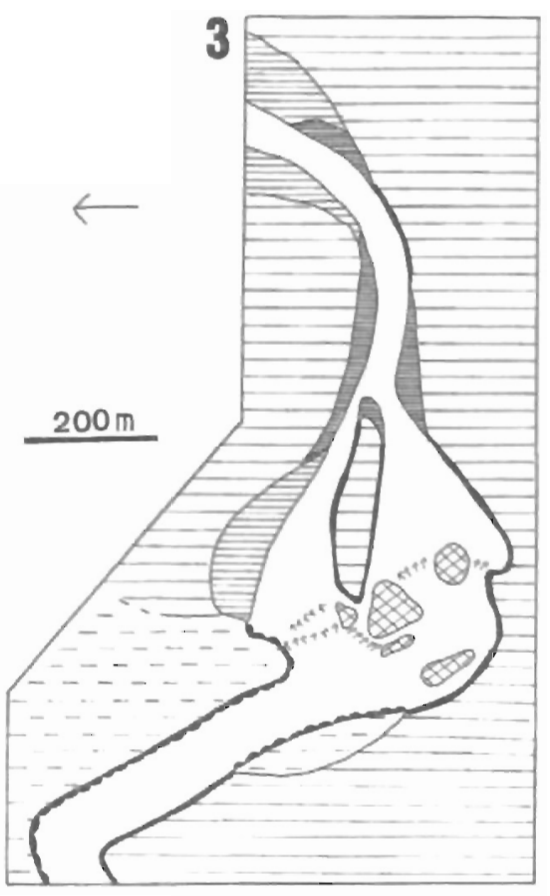

3. Saut Patawa

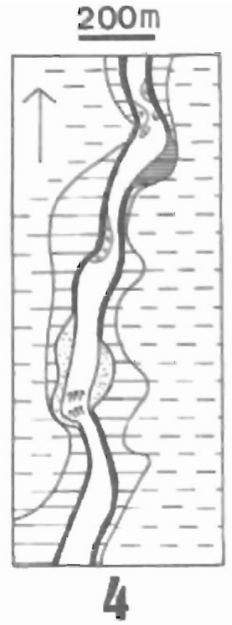

4. Saut l'Autel (dessin

B. Bordenave)

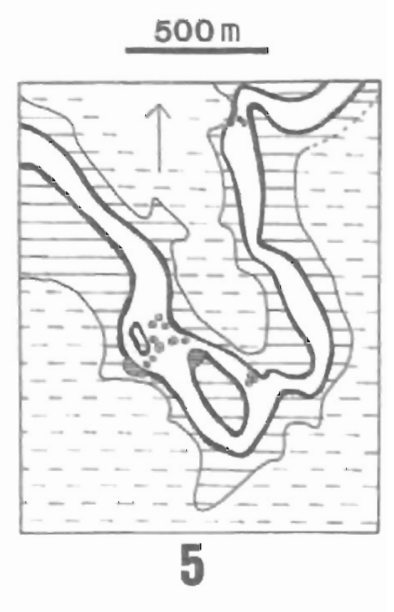

5. Saut Koumarou (dessin B. Bordenave)

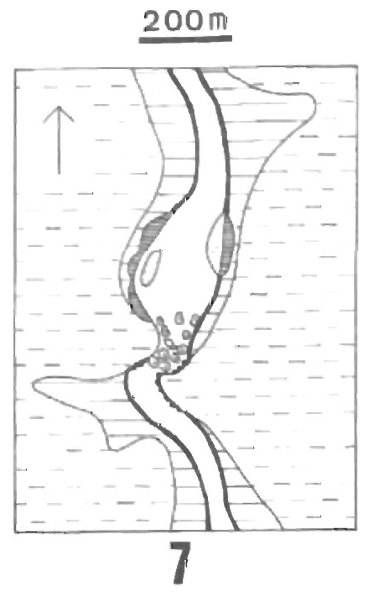

7. Saut Mouche (dessin B. Bordenave)

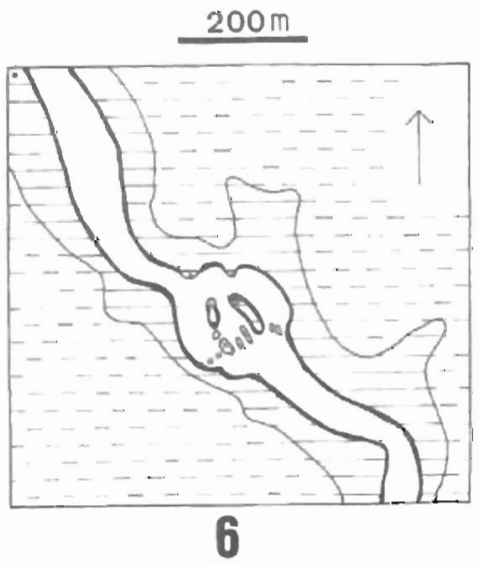

6. Saut Aimara (dessin B. Bordenave) 

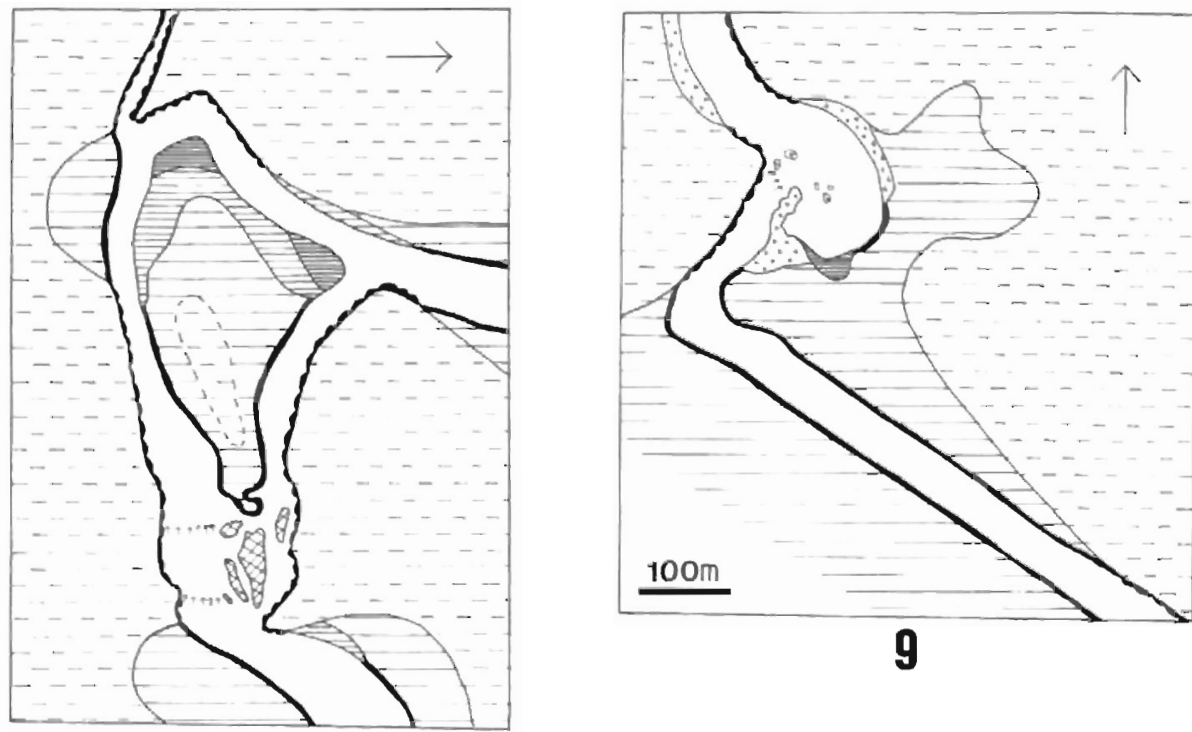

$$
100 m \quad 8
$$
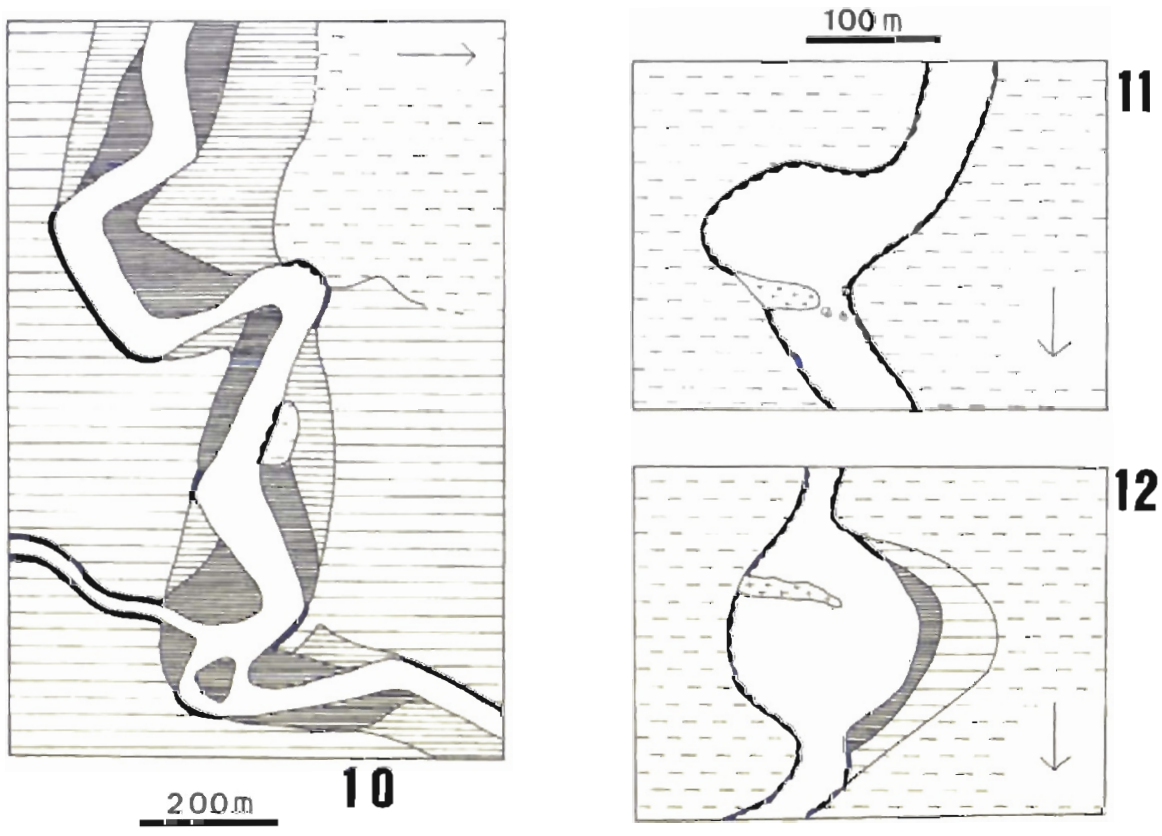

8. Saut Takari-Tanté 9. Saut Dalles

10. Saut Stéphanie

11. Saut sans nom 1

12. Saut sans nom 2 

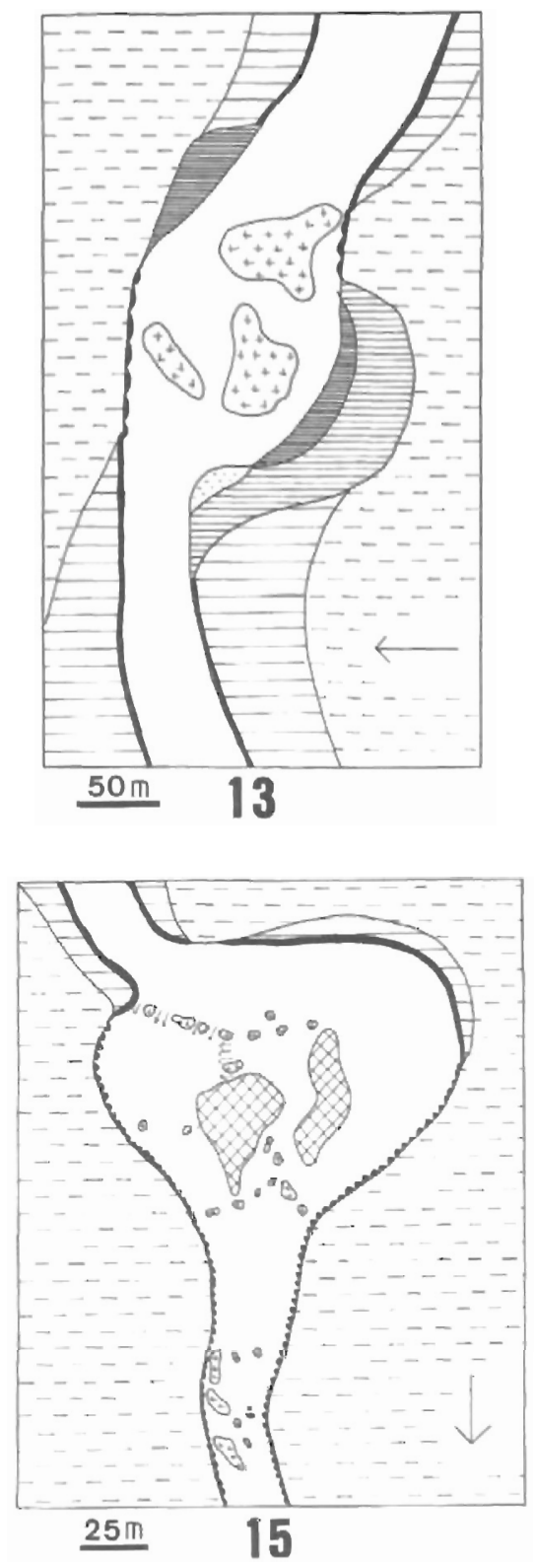
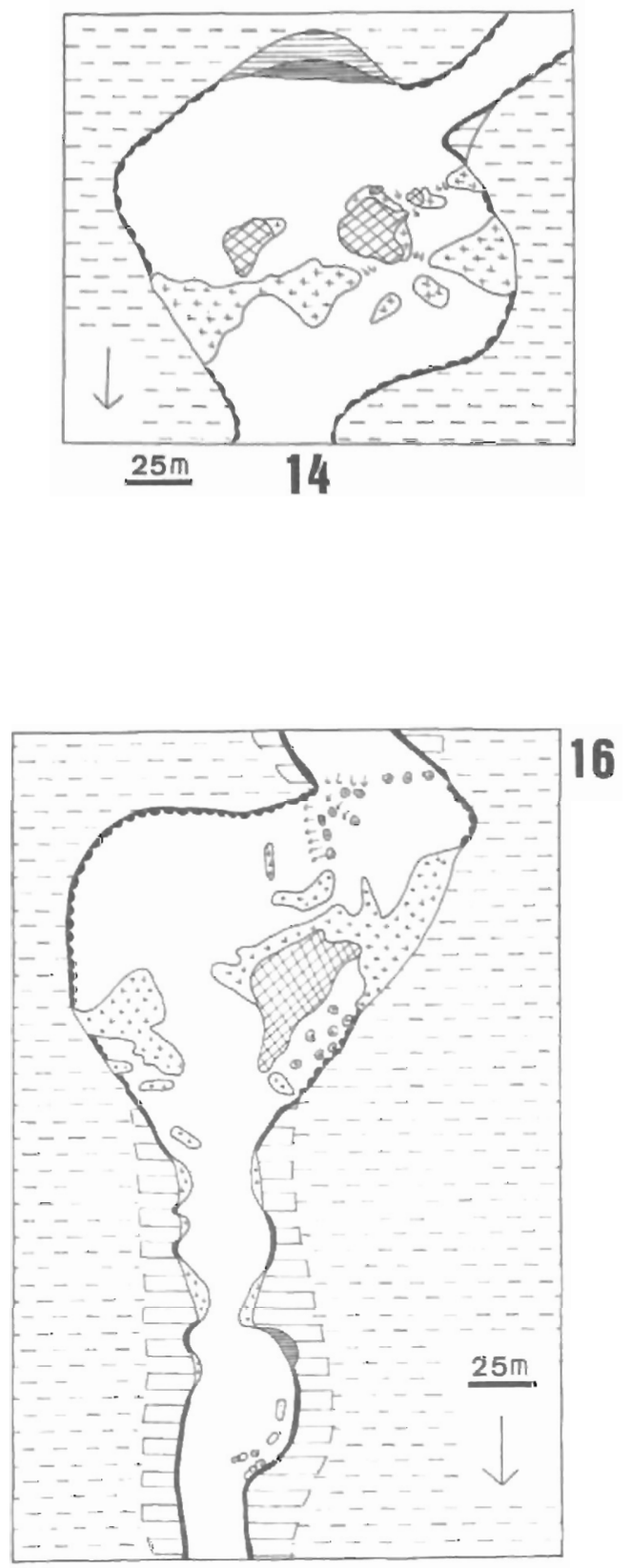

13. Saut Deux Roros 1

14. Saut Deux Roros 2

15. Saut Aristide

16. Saut Manivelle = Saut Alcide 

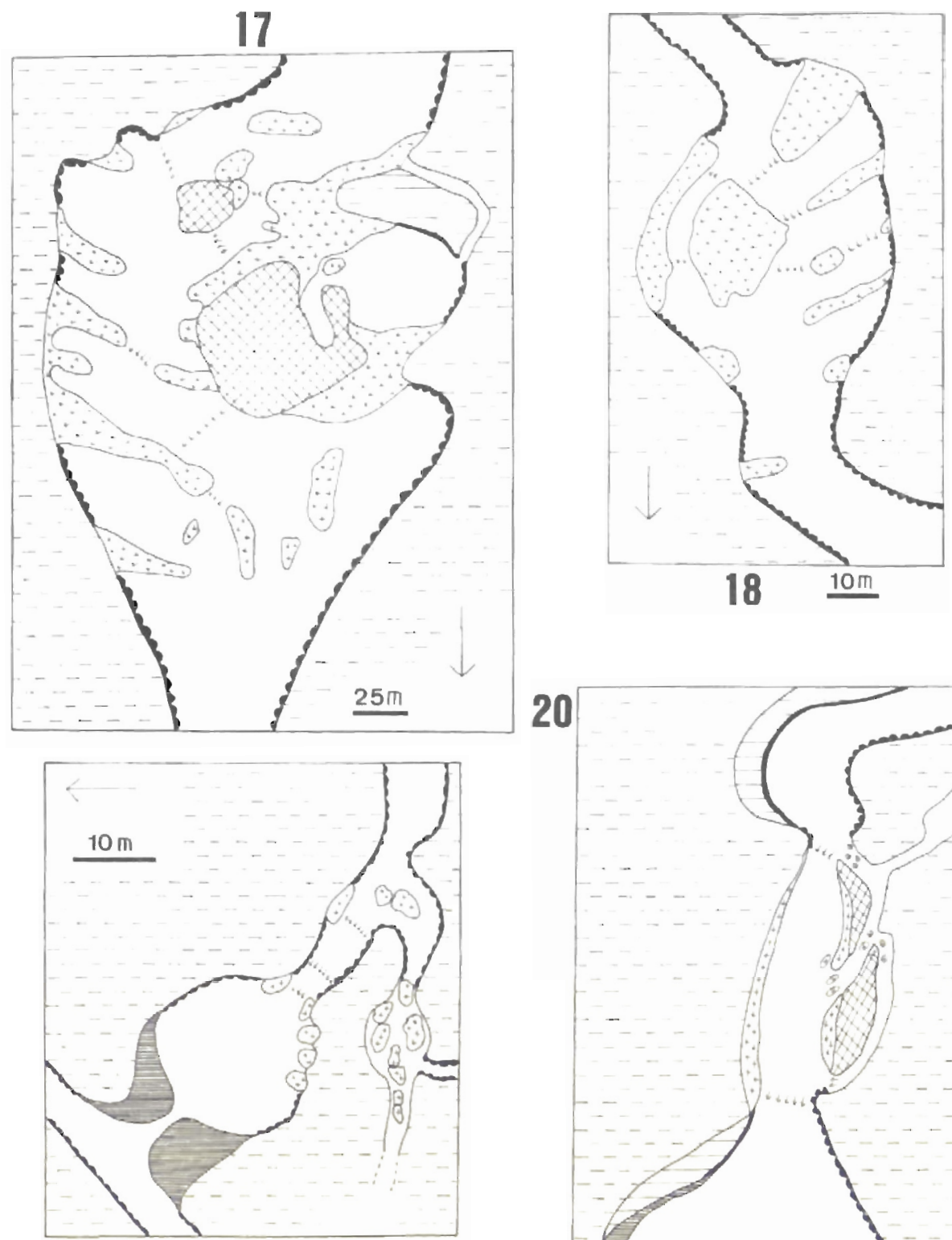

19

17. Saut du Péril

18. Saut Equerre

19. Chute Anicet

20. Saut Parasol

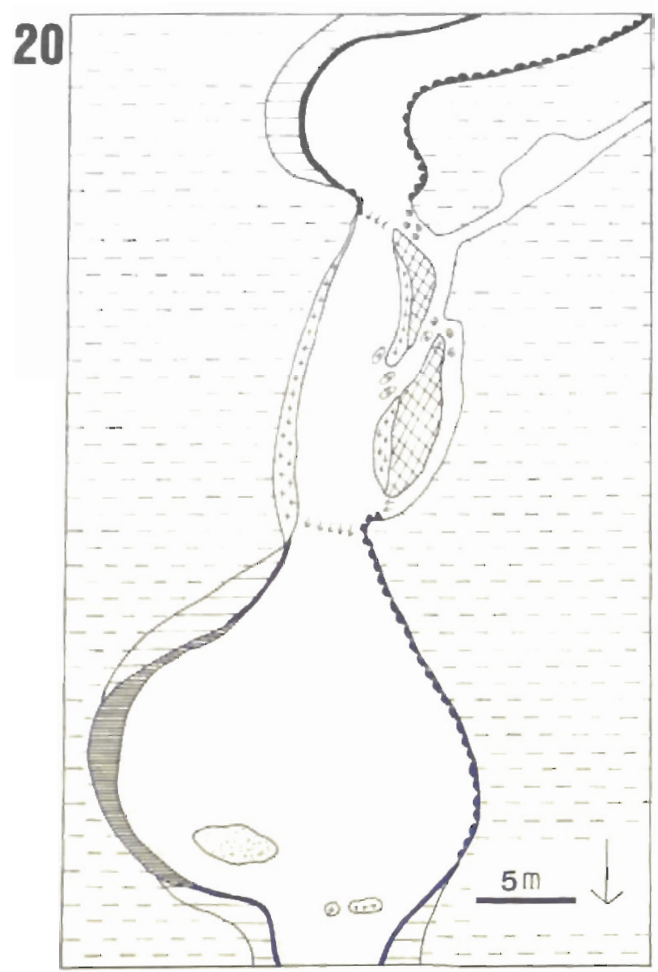




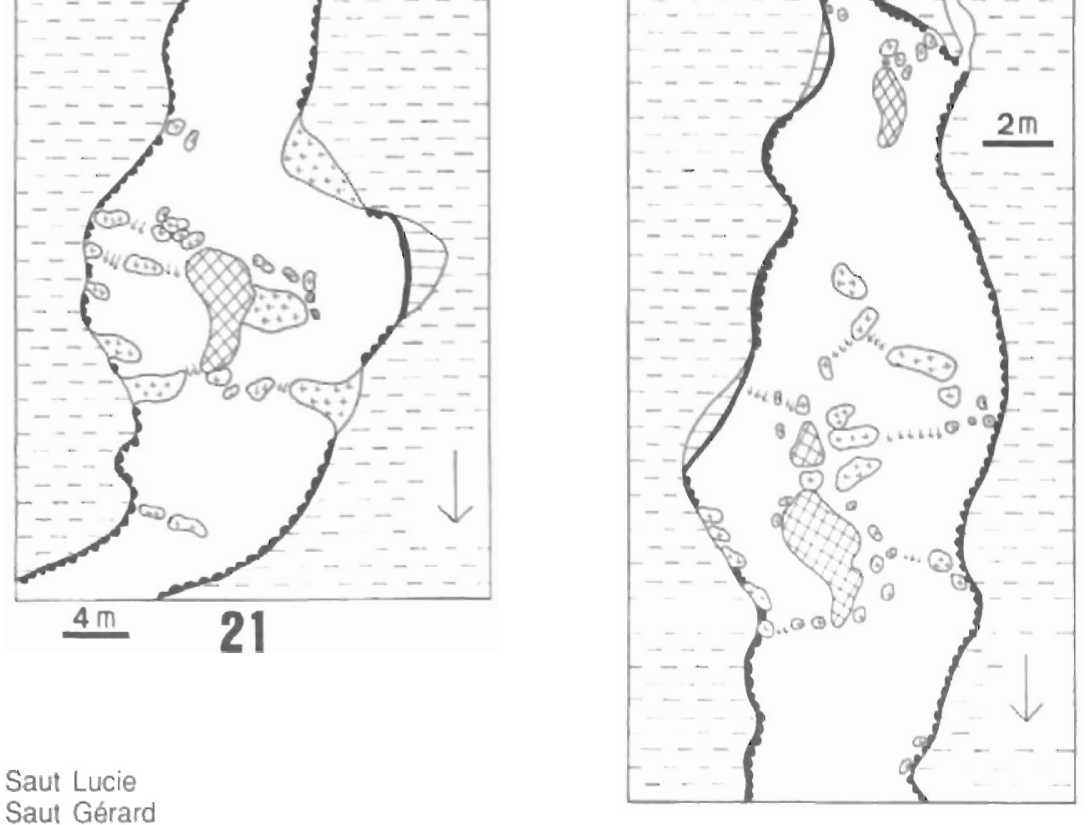

21. Saut Gérard 